IMSc/2003/04/06, CGPG-03/10-5, AEI-2003-085

\title{
Homogeneous Loop Quantum Cosmology: The Role of the Spin Connection
}

\author{
Martin Bojowald, ${ }^{1,2, *}$ Ghanashyam Date, ${ }^{3, t}$ and Kevin Vandersloot ${ }^{2, t}$ \\ ${ }^{1}$ Max-Planck-Institut für Gravitationsphysik, Albert-Einstein-Institut, \\ Am Mühlenberg 1, D-14476 Golm, Germany \\ ${ }^{2}$ Center for Gravitational Physics and Geometry, \\ The Pennsylvania State University, \\ 104 Davey Lab, University Park, PA 16802, USA \\ ${ }^{3}$ The Institute of Mathematical Sciences \\ CIT Campus, Chennai-600 113, INDIA.
}

\begin{abstract}
Homogeneous cosmological models with non-vanishing intrinsic curvature require a special treatment when they are quantized with loop quantum cosmological methods. Guidance from the full theory which is lost in this context can be replaced by two criteria for an acceptable quantization, admissibility of a continuum approximation and local stability. A quantization of the corresponding Hamiltonian constraints is presented and shown to lead to a locally stable, non-singular evolution compatible with almost classical behavior at large volume. As an application, the Bianchi IX model and its modified behavior close to its classical singularity is explored.
\end{abstract}

PACS numbers: 0460P, 0460K, 9880H

*Electronic address: mabo@aei.mpg.de

${ }^{\dagger}$ Electronic address: shyam@imsc.res.in

${ }^{\ddagger}$ Electronic address: kfvander@gravity.psu.edu 


\section{INTRODUCTION}

Since observations show that space is homogeneous at large scales to a very good approximation, homogeneous models have been studied for many decades in classical and quantum cosmology [1]. They provide insights into the behavior of our universe while avoiding all the complicated field theoretic details of full gravity by their restriction to finitely many degrees of freedom. Despite the huge reduction by infinitely many degrees of freedom, a large variety of different models is left which allow a detailed investigation of various nontrivial issues such as: Dirac observables, the embedding of symmetric models (e.g., isotropic) into less symmetric ones, dynamics, the approach to classical singularities and cosmological phenomenology.

Loop quantum cosmological methods for those models have recently been developed [2, 3, 4, 5] and shown to simplify considerably after a diagonalization of the connection and triad degrees of freedom [6]. As an example the dynamics of the Bianchi I model has been studied and shown to be singularity-free. In this case the Hamiltonian constraint resembles that of the full theory 7] (albeit it is much simpler), and thus the quantization can be regarded as a reliable test. It turned out that the extension from the isotropic case [8, 9] to this anisotropic case, requires certain features which are in fact present in quantum geometry. In particular, the mechanism of a singularity-free evolution in anisotropic models relies on the fact that the evolution extends through the classical singularity which can be identified with a submanifold in minisuperspace. This requires the classical singularity to lie in the interior of minisuperspace, rather than at the boundary (including infinity). Here it is essential that quantum geometry is based on densitized triad variables where in fact the Kasner singularity is in the interior (all densitized triad components being zero), while it would be on the infinite boundary in co-triad variables. The isotropic case is not sensitive to this issue since both densitized and co-triad variables have the classical singularity in the interior of minisuperspace (here, it is essential to use any (co-)triad rather than metric variables); thus the existence of the extension of the methods to a homogeneous model is non-trivial. An extension to the full theory of the general class of inhomogeneous models seems much more complicated at this point. However, it has been conjectured [10] that at the classical level, the most general homogeneous behavior is that of the Bianchi-IX model and furthermore that it describes the approach to the singularities even of inhomogeneous 
models. It is therefore important to study the more general Bianchi class A models.

Homogeneous models other than Bianchi I, however, present an additional complication since they have non-zero intrinsic curvature and, as a consequence, their spin connection cannot be zero. This is in contrast even to the full theory where the spin connection can be made arbitrarily small locally by choosing appropriate coordinates. In a homogeneous model the freedom of choosing coordinates is restricted to those which preserve (manifest) homogeneity, and this implies that the spin connection is a covariant object and that it has to have a certain size in a given model. This has to be taken into account properly when one quantizes the Hamiltonian constraint. In particular, in a classical regime only the extrinsic curvature can be assumed to be small, but not necessarily the intrinsic curvature which determines the spin connection. This is essential to understand the semiclassical behavior.

Since a special treatment is required which is not necessary in the full theory, one has to be more careful when interpreting the results. It introduces more quantization ambiguities which have to be shown not to influence main results. On the other hand, more possibilities for phenomenology emerge which, at least qualitatively, can often be seen to be insensitive to ambiguities.

Having a more distant relation to the full theory, it is helpful to have a set of admissibility criteria. One such criterion is of course to accommodate a semiclassical approximation to the quantum dynamics. Since a semiclassical description is based on continuum geometry while the quantum dynamics is in terms of discrete quantum geometry, this criterion is formulated here in terms of a continuum approximation. A further criterion is provided by the requirement of a locally stable evolution 11]. Such a requirement arises because the evolution equation derived along the lines of [4, 5] is usually a high order difference equation which at large volume is well approximated by the second order Wheeler-DeWitt differential equation. Hence, one can always construct solutions by choosing initial data, at large volume, to be that provided by solutions of the Wheeler-DeWitt equation. Perturbation of such an initial data will generate solutions which will also include surplus solutions of the high order difference equation. Generically these will have Planck scale oscillations. There is then the possibility that such extra solutions can become dominant under the evolution. In fact these are expected to become dominant as one gets to smaller volume since there are huge differences between the continuum and the discrete formulations in the Planck regime. However as one evolves to larger volumes, the perturbations must not grow too rapidly 
since this would imply domination by solutions with Planck scale oscillation even in the classical regime. The requirement we are looking for, local stability, prohibits this behavior by demanding that the local behavior of solutions to the difference equation around a large value of the evolution parameter is not exponentially increasing. In this paper we present a quantization of the Hamiltonian constraint for Bianchi models with non-zero intrinsic curvature which fulfills this condition and has the correct semiclassical limit. The first quantization given in [4] was not admissible in this sense; thus, the quantization given there is valid only for the Bianchi I model (for which we have the same quantization here).

It turns out that the local stability condition is selective: it requires all roots of a high order polynomial to have unit norm which is not easy to achieve randomly. The selectivity is increased by the fact that the same strategy of quantizing a Hamiltonian constraint should work in all homogeneous models, using different procedures in different models would imply that not all of them can be related to the full theory.

The stability condition can be side-stepped by quantizing connection components in the constraint by hand such that only a second order difference equation results. While this would eliminate local stability as a selection criterion, it would also imply that the quantization is even more distant to that in the full theory where such a quantization cannot be possible. Further clues as to the necessary order of the evolution equation can come from studying Dirac observables, which will be pursued elsewhere.

In section III, we briefly describe the diagonal, homogeneous models addressed in this paper. We recall the classical framework and the corresponding loop quantization, including a quantization of inverse triad components and general aspects of the Hamiltonian constraint equation. Here we also discuss the two criteria of admissibility of the continuum approximation and local stability.

In section III, we present a quantization of the Hamiltonian constraint. Since the Hamiltonian constraint now also has a potential term depending on the spin connection, a quantization of the spin connection is required. This is done using the quantization of inverse triad components. We derive the (partial) difference equation satisfied by the physical states and show that in the continuum approximation, to the leading order, the approximating differential equation is precisely the Wheeler-DeWitt equation with a specific factor ordering dictated by the underlying loop quantization. We also show that the requirement of local stability is satisfied. We demonstrate that the singularity avoidance mechanism found in 
the case of Bianchi I 6] continues to hold for these more general models. We conclude that the proposed quantization of the Hamiltonian constraint is satisfactory with respect to all our requirements.

In section IV] we focus attention on the modification of the potential implied by the quantization of the spin connection. The non-trivial and non-perturbative behavior of the inverse triad components may be expected to lead to substantial modifications of the potential at small triad components. In view of the conjectured central role of the Bianchi IX potential, we present a brief description of this potential and its implications for the modified approach to classical singularities.

\section{DIAGONAL BIANCHI CLASS A MODELS}

In the following, we restrict attention to the so called diagonal, Bianchi class A models. Among the class of homogeneous models i.e. models whose symmetry group acts transitively on the spatial manifold, is the sub-class of the Bianchi models for which the symmetry group contains a subgroup with simply transitive action on the spatial manifold. The simply transitive subgroups are classified in terms of three integers, $n^{I}$, parameterizing the structure constants as

$$
C_{J K}^{I}=\epsilon_{(I) J K} n^{I}
$$

Only class A models for which $C_{J I}^{I}=0$ admit a canonical formulation.

In the connection formulation, Bianchi class A models are described in terms of the invariant connections and densitized triads given by,

$$
A_{a}^{i}=\phi_{I}^{i} \omega_{a}^{I}, \quad E_{i}^{a}=p_{i}^{I} X_{I}^{a}
$$

with constant and canonically conjugate $\phi_{I}^{i}, p_{i}^{I}$, where $\omega^{I}$ are left-invariant (with respect to the subgroup acting simply transitively) 1-forms on the spatial manifold $\Sigma$ and $X_{I}$ are corresponding dual density-weighted vector fields. The 1-forms satisfy the Maurer-Cartan equations

$$
\mathrm{d} \omega^{I}=-\frac{1}{2} C_{J K}^{I} \omega^{J} \wedge \omega^{K}
$$

In the metric formulation, diagonal homogeneous models are those for which the spatial metric can be written in a diagonal form such that only three degrees of freedom remain. In the connection formulation the situation is analogous. To begin with one has nine degrees 
of freedom, $\phi_{I}^{i}$ but with a three parameter gauge freedom of $\mathrm{SU}(2)$ rotations of the index $i$. A further freedom, depending upon the parameters $n^{I}$, would have been available if the left-invariant 1-forms were not thought of as a background structure in parameterizing the invariant connections. To get the same three degrees of freedom as in the diagonal metric formulation, one then restricts the $\phi_{I}^{i}$ to a "diagonal form", $\phi_{I}^{i}:=c_{(I)} \Lambda_{I}^{i}$ and correspondingly

$p_{i}^{I}:=p^{I} \Lambda_{i}^{(I)}$ where the $\mathrm{SO}(3)$-matrix $\Lambda$ includes gauge degrees of freedom (see [6] for details). Such a restriction specifies the diagonal models in the connection formulation. This is not a symmetry reduction in the same sense as restriction to isotropic models is. Nevertheless, these restricted models can be analyzed by following procedures similar to those in the context of symmetry reductions. As in reductions to isotropic models, this leads to significant simplifications in such diagonalized models because the gauge parameters in $\Lambda$ and (almost; see below) gauge invariant parameters, $c_{I}$, are neatly separated [6].

\section{A. Classical Framework}

The basic variables for diagonal Bianchi Class A models are specified via

$$
A_{a}^{i}=c_{(I)} \Lambda_{I}^{i} \omega_{a}^{I}
$$

in terms of the 'gauge invariant' coefficients $c_{I}$ with the pure gauge degrees of freedom contained in the $S O(3)$-matrix $\Lambda$. The components $c_{I}$ are not completely gauge invariant but subject to residual discrete gauge transformations which change the sign of two of the three components simultaneously. A diagonal densitized triad has the form

$$
E_{i}^{a}=p^{(I)} \Lambda_{i}^{I} X_{I}^{a}
$$

where $X_{I}$ are left-invariant densitized vector fields dual to $\omega^{I}$. Being an $\mathrm{SO}(3)$-matrix, $\Lambda$ satisfies

$$
\Lambda_{I}^{i} \Lambda_{i}^{J}=\delta_{I}^{J}, \epsilon_{i j k} \Lambda_{I}^{i} \Lambda_{J}^{j} \Lambda_{K}^{k}=\epsilon_{I J K}
$$

The triad components $p^{I}$ are subject to the same residual gauge transformations as the connection components $c_{I}$ to which they are conjugate with basic Poisson bracket

$$
\left\{c_{I}, p^{J}\right\}=\gamma \kappa \delta_{I}^{J}
$$

where $\gamma$ is the Barbero-Immirzi parameter and $\kappa=8 \pi G$ the gravitational constant. We will use the value $\gamma=\frac{\log (2)}{\pi \sqrt{3}} \approx 0.13$ fixed by the black hole entropy calculations [12, 13$]$. 
A diagonal co-triad has the form $e_{a}^{i}=a_{(I)} \Lambda_{I}^{i} \omega_{a}^{I}$ with

$$
p^{1}=\left|a_{2} a_{3}\right| \operatorname{sgn}\left(a_{1}\right) \quad, \quad p^{2}=\left|a_{1} a_{3}\right| \operatorname{sgn}\left(a_{2}\right) \quad, \quad p^{3}=\left|a_{1} a_{2}\right| \operatorname{sgn}\left(a_{3}\right) .
$$

Note that the components $p^{I}$ as well as $a_{I}$ can take negative values, but only the overall sign $\operatorname{sgn}\left(p^{1} p^{2} p^{3}\right)$ (i.e., the orientation) and the absolute values $\left|p^{I}\right|$ are gauge invariant. With this form of the co-triad, we obtain, in fact, a diagonal metric

$$
\mathrm{d} s^{2}=e_{I}^{i} e_{J}^{i} \omega^{I} \omega^{J}=\sum_{I} a_{I}\left(\omega^{I}\right)^{2}
$$

The extrinsic curvature also has diagonal form with components $K_{I}=-\frac{1}{2} \dot{a}_{I}$ which appear in the relation $c_{I}=\Gamma_{I}-\gamma K_{I}$ defining the Ashtekar connection components $c_{I}$ in terms of the spin connection components $\Gamma_{I}$ and the extrinsic curvature. The form of the spin connection introduces a dependence of the framework on the particular model via the structure constants 6] :

$$
\begin{aligned}
\Gamma_{I} & =\frac{1}{2}\left(\frac{a_{J}}{a_{K}} n^{J}+\frac{a_{K}}{a_{J}} n^{K}-\frac{a_{I}^{2}}{a_{J} a_{K}} n^{I}\right) \\
& =\frac{1}{2}\left(\frac{p^{K}}{p^{J}} n^{J}+\frac{p^{J}}{p^{K}} n^{K}-\frac{p^{J} p^{K}}{\left(p^{I}\right)^{2}} n^{I}\right) \text { for }(I, J, K) \text { an even permutation of }(1,2,3) .
\end{aligned}
$$

Note that in contrast to the full theory, the spin connection is a covariant object within a homogeneous model since coordinate transformations have to respect the symmetry. Consequently, if non-zero, it cannot be made small by choosing appropriate local coordinates. The only model which has identically vanishing spin connection is the Bianchi I model with $n^{I}=0$. Otherwise, the spin connection is non-zero and can even depend on the triad (it is a constant $\Gamma=\frac{1}{2}$ in the closed isotropic model, a submodel of Bianchi IX, with $n^{I}=1$ and $\left.a_{1}=a_{2}=a_{3}\right)$.

The structure constants also appear explicitly in the Hamiltonian constraint [6]

$$
\begin{aligned}
H= & 2 \kappa^{-1}\left\{\left[\left(c_{2} \Gamma_{3}+c_{3} \Gamma_{2}-\Gamma_{2} \Gamma_{3}\right)\left(1+\gamma^{-2}\right)-n^{1} c_{1}-\gamma^{-2} c_{2} c_{3}\right] a_{1}\right. \\
& +\left[\left(c_{1} \Gamma_{3}+c_{3} \Gamma_{1}-\Gamma_{1} \Gamma_{3}\right)\left(1+\gamma^{-2}\right)-n^{2} c_{2}-\gamma^{-2} c_{1} c_{3}\right] a_{2} \\
& \left.+\left[\left(c_{1} \Gamma_{2}+c_{2} \Gamma_{1}-\Gamma_{1} \Gamma_{2}\right)\left(1+\gamma^{-2}\right)-n^{3} c_{3}-\gamma^{-2} c_{1} c_{2}\right] a_{3}\right\} \\
= & 2 \kappa^{-1}\left[\left(\Gamma_{2} \Gamma_{3}-n^{1} \Gamma_{1}\right) a_{1}+\left(\Gamma_{1} \Gamma_{3}-n^{2} \Gamma_{2}\right) a_{2}+\left(\Gamma_{1} \Gamma_{2}-n^{3} \Gamma_{3}\right) a_{3}\right. \\
& \left.-\frac{1}{4}\left(a_{1} \dot{a}_{2} \dot{a}_{3}+a_{2} \dot{a}_{1} \dot{a}_{3}+a_{3} \dot{a}_{1} \dot{a}_{2}\right)\right] .
\end{aligned}
$$


In order to derive the classical field equations (for which we consider only positive $a_{I}$ and $\left.p^{I}\right)$ it is advantageous to transform to new canonical variables

$$
\begin{array}{lll}
\pi_{I}:=2 K_{I} p^{(I)}=-\dot{a}_{I} a_{I}^{-1} a_{1} a_{2} a_{3}=-\left(\log a_{I}\right)^{\prime} & \text { and } \\
q^{I}:=\frac{1}{2} \log p^{I} & \text { such that } \quad & \left\{q^{I}, \pi_{J}\right\}=\kappa \delta_{I}^{J}
\end{array}
$$

where the prime denotes a derivative with respect to a new time coordinate $\tau$ related to $t$ by $\mathrm{d} t=a_{1} a_{2} a_{3} \mathrm{~d} \tau$ (corresponding to the lapse function $N=a_{1} a_{2} a_{3}$ ). With these new variables we have $\left\{\pi_{I}, p^{J}\right\}=-2 \kappa p^{(I)} \delta_{I}^{J}$ and

$$
\begin{aligned}
\kappa N H= & \kappa a_{1} a_{2} a_{3} H=2\left[p^{1} p^{2}\left(\Gamma_{1} \Gamma_{2}-n^{3} \Gamma_{3}\right)+p^{1} p^{3}\left(\Gamma_{1} \Gamma_{3}-n^{2} \Gamma_{2}\right)+p^{2} p^{3}\left(\Gamma_{2} \Gamma_{3}-n^{1} \Gamma_{1}\right)\right. \\
& \left.-\frac{1}{4}\left(\pi_{1} \pi_{2}+\pi_{1} \pi_{3}+\pi_{2} \pi_{3}\right)\right] \\
= & \frac{1}{2}\left[\left(n^{1}\right)^{2}\left(\frac{p^{2} p^{3}}{p^{1}}\right)^{2}+\left(n^{2}\right)^{2}\left(\frac{p^{1} p^{3}}{p^{2}}\right)^{2}+\left(n^{3}\right)^{2}\left(\frac{p^{1} p^{2}}{p^{3}}\right)^{2}\right] \\
& -n^{1} n^{2}\left(p^{3}\right)^{2}-n^{1} n^{3}\left(p^{2}\right)^{2}-n^{2} n^{3}\left(p^{1}\right)^{2}-\frac{1}{2}\left(\pi_{1} \pi_{2}+\pi_{1} \pi_{3}+\pi_{2} \pi_{3}\right) \\
= & \frac{1}{2}\left[\left(n^{1}\right)^{2} a_{1}^{4}+\left(n^{2}\right)^{2} a_{2}^{4}+\left(n^{3}\right)^{2} a_{3}^{4}\right]-n^{1} n^{2} a_{1}^{2} a_{2}^{2}-n^{1} n^{3} a_{1}^{2} a_{3}^{2}-n^{2} n^{3} a_{2}^{2} a_{3}^{2} \\
& -\frac{1}{2}\left(\pi_{1} \pi_{2}+\pi_{1} \pi_{3}+\pi_{2} \pi_{3}\right) .
\end{aligned}
$$

Now one can easily derive the equations of motion,

$$
\left(\log a_{I}\right)^{\prime \prime}=-\pi_{I}^{\prime} \approx-\left\{\pi_{I}, a_{1} a_{2} a_{3} H\right\}
$$

which yield

$$
\begin{aligned}
& \frac{1}{2}\left(\log a_{1}\right)^{\prime \prime}=\left(n^{2} a_{2}^{2}-n^{3} a_{3}^{2}\right)^{2}-\left(n^{1}\right)^{2} a_{1}^{4} \\
& \frac{1}{2}\left(\log a_{2}\right)^{\prime \prime}=\left(n^{1} a_{1}^{2}-n^{3} a_{3}^{2}\right)^{2}-\left(n^{2}\right)^{2} a_{2}^{4} \\
& \frac{1}{2}\left(\log a_{3}\right)^{\prime \prime}=\left(n^{1} a_{1}^{2}-n^{2} a_{2}^{2}\right)^{2}-\left(n^{3}\right)^{2} a_{3}^{4}
\end{aligned}
$$

or, using $\left(q^{1}\right)^{\prime \prime}=-\frac{1}{2}\left(\pi_{2}^{\prime}+\pi_{3}^{\prime}\right)$

$$
\left(q^{I}\right)^{\prime \prime}=-4 a_{I}^{2} p^{I} \Gamma_{I} n^{I}
$$

For the Bianchi I model we have $n^{I}=0$ so that $\log a_{I}=\alpha_{I}\left(\tau-\tau_{0, I}\right)$. This implies the Kasner behavior $a_{I} \propto t^{\alpha_{I}}$ where $t=e^{\tau}$ and the constraint requires $0=\alpha_{1} \alpha_{2}+\alpha_{1} \alpha_{3}+\alpha_{2} \alpha_{3}=$ $\frac{1}{2}\left(\left(\alpha_{1}+\alpha_{2}+\alpha_{3}\right)^{2}-\alpha_{1}^{2}-\alpha_{2}^{2}-\alpha_{3}^{2}\right)$. The coefficients $\alpha_{I}$ can be rescaled by choosing a different $t(\tau)$ which can be fixed by requiring the conventional parameterization $\alpha_{1}+\alpha_{2}+\alpha_{3}=1=$ $\alpha_{1}^{2}+\alpha_{2}^{2}+\alpha_{3}^{2}$. As usual, these equations can be solved only if one coefficient, say $\alpha_{1}$, is negative 
and the other two are positive. Correspondingly, one direction, the first, contracts whereas the other two expand toward larger time. When we approach the classical singularity at $t=0$, space shrinks only in two directions while the third one expands unboundedly. The total volume however continues to approach zero according to $a_{1} a_{2} a_{3} \propto t^{\alpha_{1}+\alpha_{2}+\alpha_{3}}=t$. In 6] it has been shown that the Kasner singularity disappears when the model is quantized along the lines of loop quantum cosmology. For this result it was important that quantum geometry is based on densitized triad variables rather than co-triad variables. In those variables, all $p^{I} \propto t^{1-\alpha_{I}}$ decrease to zero when one approaches the classical singularity. By contrast, for the minisuperspace described in terms of the scale factors or co-triad variables $\left(a_{I}\right)$, the singularity is reached when at least one of them goes to infinity. We paraphrase this by saying that the classical singularity lies in the interior of the triad minisuperspace (containing triads of both orientations and also degenerate ones).

Bianchi models other than Bianchi I have non-vanishing structure constants and thus the evolution of the three triad components can be described as motion in a non-trivial potential given by

$$
\begin{aligned}
W\left(p^{1}, p^{2}, p^{3}\right) & =2\left\{p^{1} p^{2}\left(\Gamma_{1} \Gamma_{2}-n^{3} \Gamma_{3}\right)+p^{1} p^{3}\left(\Gamma_{1} \Gamma_{3}-n^{2} \Gamma_{2}\right)+p^{2} p^{3}\left(\Gamma_{2} \Gamma_{3}-n^{1} \Gamma_{1}\right)\right\} \\
& =\left(p^{1}\right)^{2} \Gamma_{1}^{2}+\left(p^{2}\right)^{2} \Gamma_{2}^{2}+\left(p^{3}\right)^{2} \Gamma_{3}^{2}-\left(p^{1} \Gamma_{1}+p^{2} \Gamma_{2}+p^{3} \Gamma_{3}\right)^{2}
\end{aligned}
$$

which has infinite walls at small $p^{I}$ owing to the divergence of the spin connection components (see Fig. 2). The evolution can then be described approximately as a succession of Kasner epochs with intermediate reflections at the potential [14]. For the Bianchi IX model the reflections never stop and the classical evolution is believed to be chaotic [15].

\section{B. Loop Quantization}

Diagonal homogeneous loop quantum cosmology [6] is first formulated in the connection representation where an orthonormal basis is given by the $\hat{p}^{I}$-eigenstates

$$
\left|m_{1}, m_{2}, m_{3}\right\rangle:=\left|m_{1}\right\rangle \otimes\left|m_{2}\right\rangle \otimes\left|m_{3}\right\rangle
$$

with

$$
\langle c \mid m\rangle=\frac{\exp \left(\frac{1}{2} i m c\right)}{\sqrt{2} \sin \left(\frac{1}{2} c\right)}
$$


(The full Hilbert space of loop quantum cosmology is non-separable, but for our purposes it is sufficient to use the separable subspace spanned by the states (15); see [16] for a detailed discussion.) The eigenvalues of the triad operators can be read off from

$$
\hat{p}^{I}\left|m_{1}, m_{2}, m_{3}\right\rangle=\frac{1}{2} \gamma \ell_{\mathrm{P}}^{2} m_{I}\left|m_{1}, m_{2}, m_{3}\right\rangle
$$

Using the basic operators $\hat{p}^{I}$ one can define the volume operator $\hat{V}=\sqrt{\left|\hat{p}^{1} \hat{p}^{2} \hat{p}^{3}\right|}$ which will be used later. Its eigenstates are also $\left|m_{1}, m_{2}, m_{3}\right\rangle$ with eigenvalues

$$
V\left(m_{1}, m_{2}, m_{3}\right)=\left(\frac{1}{2} \gamma \ell_{\mathrm{P}}^{2}\right)^{\frac{3}{2}} \sqrt{\left|m_{1} m_{2} m_{3}\right|} .
$$

A kinematical state $|s\rangle$ is described in the triad representation by coefficients $s_{m_{1}, m_{2}, m_{3}}$ defined via,

$$
|s\rangle=\sum_{m_{1}, m_{2}, m_{3}} s_{m_{1}, m_{2}, m_{3}}\left|m_{1}, m_{2}, m_{3}\right\rangle
$$

For a state to be gauge invariant under the residual gauge transformations, the coefficients $s_{m_{1}, m_{2}, m_{3}}$ have to satisfy

$$
s_{m_{1}, m_{2}, m_{3}}=s_{-m_{1},-m_{2}, m_{3}}=s_{m_{1},-m_{2},-m_{3}}=s_{-m_{1}, m_{2},-m_{3}} .
$$

These states are left invariant by the gauge invariant triad operators $\left|\hat{p}^{I}\right|$ and the orientation operator $\operatorname{sgn}\left(\hat{p}^{1} \hat{p}^{2} \hat{p}^{3}\right)$. In calculations it is often easier to work with non-gauge invariant states in intermediate steps and project to gauge invariant ones in the end.

Together with the basic derivative operators $\hat{p}^{I}$ we need multiplication operators which usually arise from (point) holonomies $h_{I}=\exp \left(c_{(I)} \Lambda_{I}^{i} \tau_{i}\right)=\cos \left(\frac{1}{2} c_{I}\right)+2 \Lambda_{I}^{i} \tau_{i} \sin \left(\frac{1}{2} c_{I}\right)$ with action

$$
\begin{aligned}
\cos \left(\frac{1}{2} c_{1}\right)\left|m_{1}, m_{2}, m_{3}\right\rangle & =\frac{1}{2}\left(\left|m_{1}+1, m_{2}, m_{3}\right\rangle+\left|m_{1}-1, m_{2}, m_{3}\right\rangle\right) \\
\sin \left(\frac{1}{2} c_{1}\right)\left|m_{1}, m_{2}, m_{3}\right\rangle & =-\frac{1}{2} i\left(\left|m_{1}+1, m_{2}, m_{3}\right\rangle-\left|m_{1}-1, m_{2}, m_{3}\right\rangle\right)
\end{aligned}
$$

and correspondingly for $c_{2}$ and $c_{3}$.

\section{Inverse triad operators}

From the basic operators we can build more complicated ones. We will later need a quantization of the spin connection which is a composite operator containing several triad 
operators. In particular, it also contains inverse powers of triad components which classically diverge at the singularity. Since the triad operators have a discrete spectrum containing zero, they do not have an inverse. However, general methods of quantum geometry and loop quantum cosmology [17, 18] imply that there exist well-defined operators quantizing inverse triad components. To obtain these operators one makes use of a classical reformulation, e.g.

$$
\left|p^{1}\right|^{-1}=4 \gamma^{-2} \kappa^{-2}\left(\left\{c_{1}, \sqrt{\left|p^{1}\right|}\right\}\right)^{2}=6 \gamma^{-2} \kappa^{-2}\left(\operatorname{tr} \Lambda_{1}^{i} \tau_{i} h_{I}\left\{h_{I}^{-1}, \sqrt{\left|p^{1}\right|}\right\}\right)^{2}
$$

which can then be quantized to

$$
\widehat{\left|p^{1}\right|_{j}^{-1}}=-36 \gamma^{-2} \ell_{\mathrm{P}}^{-4}[j(j+1)(2 j+1)]^{-2}\left(\operatorname{tr}_{j} \Lambda_{1}^{i} \tau_{i} h_{1}\left[h_{1}^{-1}, \sqrt{\left|\hat{p}^{1}\right|}\right]\right)^{2} \text {. }
$$

Here we have indicated that there are quantization ambiguities [19] when one quantizes composite operators. The one, most relevant for this paper, is indicated by the subscript $j$ of the trace and corresponds to the choice of representation while writing holonomies as multiplicative operators.

This operator acts as

$$
\begin{aligned}
& \widehat{\left|p^{I}\right|_{j}^{-1}}\left|m_{1}, m_{2}, m_{3}\right\rangle:=\left(\frac{1}{2} \gamma \ell_{p}^{2}\right)^{-1} \mathcal{N}_{j}^{-2} f_{j}\left(m_{I}\right)\left|m_{1}, m_{2}, m_{3}\right\rangle \quad \text { where, } \\
& f_{j}\left(m_{I}\right):=\left\{\sum_{k=-j}^{j} k \sqrt{\left|m_{I}+2 k\right|}\right\}^{2} \quad \text { and } \\
& \mathcal{N}_{j}:=\frac{j(j+1)(2 j+1)}{3} \text {. }
\end{aligned}
$$

The discrete values $f_{j}(m)$ decrease toward lower values for $m<2 j$ [19]. Thus, one can see that the classical divergence of the inverse of $\left|p^{I}\right|$ at vanishing $p^{I}$ is explicitly absent in the quantized operator. This will be seen to have further consequences for the approach to the classical singularity. Furthermore, the state $\left|m_{1}, m_{2}, m_{3}\right\rangle$ with $m_{I}=0$ (on which the classical inverse triad would diverge) is actually annihilated by $\widehat{\left|p^{I}\right|_{j}^{-1}}$ due to $f_{j}(0)=0$. This allows us to define the inverse triad operator (not just its absolute value) by $\widehat{\left(p^{I}\right)_{j}^{-1}}:=\operatorname{sgn}\left(\hat{p}^{I}\right) \widehat{\left|p^{I}\right|_{j}^{-1}}$ without ambiguity in defining the sign of zero. These inverse triad operators will be used later to find well-defined operators quantizing the spin connection. Their eigenvalues are

$$
\widehat{\left(p^{I}\right)_{j}^{-1}}\left|m_{1}, m_{2}, m_{3}\right\rangle=\left(\frac{1}{2} \gamma \ell_{p}^{2}\right)^{-1} \mathcal{N}_{j}^{-2} \operatorname{sgn}\left(m_{I}\right) f_{j}\left(m_{I}\right)\left|m_{1}, m_{2}, m_{3}\right\rangle
$$




\section{The Hamiltonian constraint}

Since the classical Hamiltonian constraint contains the structure constants, it must have a model dependent quantization. The procedure in the full theory 7] motivates to model the holonomy of a closed loop by using the combination $h_{I} h_{J} h_{I}^{-1} h_{J}^{-1}$ in order to quantize connection components in the constraint [4]. This works in fact for the Bianchi I model, but it must be modified for models with non-vanishing intrinsic curvature. In these models a curve which is formed from four pieces of integral curves of vector fields $X_{I}$ generating the symmetry action, which would have holonomy $h_{I} h_{J} h_{I}^{-1} h_{J}^{-1}$, does not close to form a loop. One needs a correction which in [4] has been done by adding a holonomy for the remaining curve multiplicatively. However this does not result in an admissible evolution for the closed isotropic model derived from Bianchi IX 11] (an admissible quantization for this model has recently been presented in [20]). We thus have to find a quantization which satisfies certain criteria for a well-defined evolution.

To appreciate the criteria we recall that the general form of the Hamiltonian constraint yields a difference equation for the wave function in the triad representation because of the action of holonomy operators [5]. This fact is directly related to the discreteness of quantum geometry. However in the classical regime of large volume and small extrinsic curvature, the geometry is the familiar continuum (metric) geometry. Thus the difference equation must admit solutions which can be very well approximated by solutions of differential equations, namely the Wheeler-DeWitt equation which comes from the (Schrodinger) quantization of continuum geometry. Such solutions are slowly varying (in the classical regime) and can be interpolated by slowly varying solutions of the Wheeler-DeWitt equation and are termed as pre-classical. Since quantum geometry depends on the Barbero-Immirzi parameter while the continuum geometry does not, absence of $\gamma$ dependence in arriving at the differential approximation provides non-trivial constraints on the coefficients of the difference equation. Admissibility of a continuum approximation is our first criterion.

However, admissibility of a continuum approximation alone is not enough. Typically the order of the difference equation is larger than that of the Wheeler-DeWitt equation. Consequently there are solutions of the difference equation which are not interpolable by solutions of differential equation. Therefore, if in some local region in the classical regime we have a "small" admixture of these extra solutions (small so that the continuum approximation is 
valid), there is no guarantee that contribution of the extra solutions remains small under subsequent evolution, i.e. the continuum approximation may get invalidated. That this does not happen is our second criterion. It is thus necessary for the stability of the continuum approximation.

A detailed formulation of these criteria is given in [11]. As a first step, one divides the classical regime (large eigenvalues of the triad operators) into smaller cells (local regions) which are still large compared to the order of the difference equation. In each of these cells, the coefficients of the difference equations are approximately constant. This leads to approximating the difference equation by an equation with constant coefficients. In the case of ordinary difference equations that result in the isotropic models, the solutions are controlled by the roots of a polynomial. The admissibility of continuum approximation puts a set of conditions on these constant coefficients which translate into conditions on the roots. For the isotropic case in particular, the criterion requires existence of a root with value 1 and multiplicity 2. The second criterion of local stability further requires that all roots must have absolute value less than or equal to 1 in order to prevent exponential growth of the non-pre-classical solutions. A further complex conjugation property of the coefficients of the difference equations then implies that all roots in fact must have absolute value 1 .

The quantization of the Hamiltonian constraint presented in the next section will be shown to satisfy both these criteria.

\section{HAMILTONIAN CONSTRAINTS FOR BIANCHI CLASS A MODELS}

As noted in the introduction, models with non-vanishing intrinsic curvature must have non-zero spin connection which, thanks to homogeneity, cannot be made to vanish. Therefore, the Ashtekar connection, a linear combination of the spin connection and the extrinsic curvature, is not necessarily small even in a semiclassical regime corresponding to small extrinsic curvature. While the Hamiltonian constraint in quantum geometry is formulated naturally in terms of the connection, for a semiclassical approximation it is desirable to perform an expansion in the small extrinsic curvature. This can only lead to the correct result if the spin connection also appears explicitly in the constraint. We will first propose the quantized constraint operator, show how it reproduces the classical expression and heuristi-

cally argue how it can admit a continuum approximation with local stability. The properties 
of the proposed operator will be elaborated in detail in the following subsections.

The proposed Hamiltonian constraint operator is:

$$
\begin{aligned}
\hat{H}=4 i\left(\gamma \ell_{p}^{2} \kappa\right)^{-1} \sum_{I J K} \epsilon^{I J K} \operatorname{tr}[ & \left\{\gamma^{-2} \hat{h}_{I}(A, \Gamma) \hat{h}_{J}(A, \Gamma)\left(\hat{h}_{I}(A, \Gamma)\right)^{-1}\left(\hat{h}_{J}(A, \Gamma)\right)^{-1}\right. \\
& \left.\left.-2\left(\hat{\Gamma}_{I} \hat{\Gamma}_{J}-n^{L} \hat{\Gamma}_{L}\right) \Lambda_{I} \Lambda_{J}\right\}\left\{h_{K}(A)\left[h_{K}^{-1}(A), \hat{V}\right]\right\}\right]
\end{aligned}
$$

where

$$
\begin{aligned}
\hat{h}_{I}(A, \Gamma) & :=e^{c_{(I)} \Lambda_{I}^{i} \tau_{i}} e^{-\hat{\Gamma}_{(I)} \Lambda_{I}^{i} \tau_{i}} \\
\hat{\Gamma}_{I} & :=\frac{1}{2}\left[\hat{p}^{J} n^{K} \widehat{\left(p^{K}\right)^{-1}}+\hat{p}^{K} n^{J} \widehat{\left(p^{J}\right)^{-1}}-\hat{p}^{J} \hat{p}^{K} n^{I}\left(\widehat{\left(p^{I}\right)^{-1}}\right)^{2}\right]
\end{aligned}
$$

and the $\widehat{\left(p^{I}\right)^{-1}}$ are defined in equation (25). Since the inverse triad operators commute with the triad operators, there are no ordering ambiguities in the spin connection (28). Note that the holonomy operators $h_{K}(A)=\exp \left(c_{(K)} \Lambda_{K}^{i} \tau_{i}\right)$ appearing in the second set of braces do not need to contain the spin connection since it commutes with $\hat{V}$ and would drop out.

In (26) the quantized spin connection appears explicitly both in the diagonal 'potential' term and in holonomies $h_{I}(A, \Gamma)$. For vanishing $\Gamma_{I}$ the constraint operator reproduces the one of the Bianchi-I model [4, 6]. It is easy to see that the term $\left(\Gamma_{I} \Gamma_{J}-n^{L} \Gamma_{L}\right) \Lambda_{I} \Lambda_{J}$ comes from the curvature 2 -form of the spin connection $\Gamma$ for a Bianchi model. In fact, an expression via the curvature components would be more general since it could also be used for Kantowski-Sachs models.

The holonomies $h_{I}(A, \Gamma)$ are products of holonomies $h_{I}(A)$ and $\left(h_{I}(\Gamma)\right)^{-1}$. Since we have to choose a quantization of the spin connection components $\Gamma_{I}$, which contain inverse triads, and also an ordering of the non-commuting $A$ - and $\Gamma$-holonomies, there are many more quantization ambiguities compared to the Bianchi-I model. However, qualitative effects can often be seen to be independent of such ambiguities.

To see that the proposed Hamiltonian operator goes over to the classical expression in the classical limit, we can ignore the fact that connection components and the spin connection do not commute since this will only lead to effects of the order $\hbar$ which disappear in the classical limit. The holonomy product $h_{I}(A) h_{I}(\Gamma)^{-1}$ can then be written formally as

$$
h_{I}(A) h_{I}(\Gamma)^{-1} \sim \cos \left(\frac{1}{2}\left(c_{I}-\Gamma_{I}\right)\right)+2 \Lambda_{I}^{i} \tau_{i} \sin \left(\frac{1}{2}\left(c_{I}-\Gamma_{I}\right)\right)
$$

which for small $K_{I}=-\gamma^{-1}\left(c_{I}-\Gamma_{I}\right)$ yields

$$
h_{I}(A) h_{I}(\Gamma)^{-1} \sim 1-2 \gamma \Lambda_{I}^{i} \tau_{i} K_{I} .
$$


On states (15) with large labels $m_{I}$, the commutator containing the volume operator turns into

$$
h_{K}\left[h_{K}^{-1}, \hat{V}\right] \sim-\frac{1}{2} i \gamma \ell_{\mathrm{P}}^{2} \Lambda_{K} a_{K}
$$

so that the classical constraint in the form (7) is reproduced.

With regard to the continuum approximation and the local stability conditions, we have to take into account the fact that the connection does not commute with the spin connection because even small terms can have significant effects should they lead to instabilities. The products of holonomies then do not depend on the differences $c_{I}-\Gamma_{I}$ only, as could be expected if they were to commute. While ignoring the non-commutativity we have seen that expressions like $\sin \left(\frac{1}{2}\left(c_{I}-\Gamma_{I}\right)\right)$ appear which, however, are not well-defined in this form since there do not exist $c_{I^{-}}$operators in quantum geometry (only holonomies of the Ashtekar connection are well-defined operators). Motivated by the form of the holonomies in (27),

$$
\begin{aligned}
h_{I}(A, \Gamma) & =\left(\cos \left(\frac{1}{2} c_{I}\right)+2 \Lambda_{I} \sin \left(\frac{1}{2} c_{I}\right)\right)\left(\cos \left(\frac{1}{2} \hat{\Gamma}_{I}\right)-2 \Lambda_{I} \sin \left(\frac{1}{2} \hat{\Gamma}_{I}\right)\right) \\
& =\frac{1}{2}\left(e^{\frac{1}{2} i c_{I}} e^{-\frac{1}{2} i \hat{\Gamma}_{I}}+e^{-\frac{1}{2} i c_{I}} e^{\frac{1}{2} i \hat{\Gamma}_{I}}-2 i \Lambda_{I}\left(e^{\frac{1}{2} i c_{I}} e^{-\frac{1}{2} i \hat{\Gamma}_{I}}-e^{-\frac{1}{2} i c_{I}} e^{\frac{1}{2} i \hat{\Gamma}_{I}}\right)\right)
\end{aligned}
$$

we define $\widehat{\sin }$ and $\widehat{\cos }$ operators by

$$
h_{I}(A, \Gamma)=: \widehat{\cos }\left(\frac{1}{2}\left(c_{I}-\Gamma_{I}\right)\right)+2 \Lambda_{I} \widehat{\sin }\left(\frac{1}{2}\left(c_{I}-\Gamma_{I}\right)\right)
$$

with action

$$
\begin{aligned}
\widehat{\sin }\left(\frac{1}{2}\left(c_{1}-\Gamma_{1}\right)\right)\left|m_{1}, m_{2}, m_{3}\right\rangle:= & -\frac{1}{2} i\left(e^{\frac{1}{2} i c_{1}} e^{-\frac{1}{2} i \Gamma_{1}}-e^{-\frac{1}{2} i c_{1}} e^{\frac{1}{2} i \Gamma_{1}}\right)\left|m_{1}, m_{2}, m_{3}\right\rangle \\
= & -\frac{1}{2} i\left(e^{-\frac{1}{2} i \Gamma_{1}\left(m_{I}\right)}\left|m_{1}+1, m_{2}, m_{3}\right\rangle\right. \\
& \left.-e^{\frac{1}{2} i \Gamma_{1}\left(m_{I}\right)}\left|m_{1}-1, m_{2}, m_{3}\right\rangle\right)
\end{aligned}
$$

and analogously for $\widehat{\mathrm{COS}}-\mathrm{operators.} \mathrm{This} \mathrm{means} \mathrm{that,} \mathrm{by} \mathrm{definition,} \mathrm{the} \mathrm{spin} \mathrm{connection}$ in trigonometric expressions of $c_{I}-\Gamma_{I}$, always acts first. In the final expression we used eigenvalues $\Gamma_{1}\left(m_{I}\right)$ of the spin connection operators,

$$
\Gamma_{J}\left|m_{1}, m_{2}, m_{3}\right\rangle:=\Gamma_{J}\left(m_{I}\right)\left|m_{1}, m_{2}, m_{3}\right\rangle
$$

Operators of the form (29) however, do not produce the basic difference operator in the triad representation since they contain the spin connection eigenvalues as coefficients. 
Nevertheless, for large $m_{I}$ we have in the triad representation for a state $|s\rangle$

$$
\begin{aligned}
\left(\widehat{\sin }\left(\frac{1}{2}\left(c_{1}-\Gamma_{1}\right)\right) s\right)_{m_{1}, m_{2}, m_{3}} & =\frac{1}{2} i\left(e^{\frac{1}{2} i \Gamma_{1}\left(m_{1}+1, m_{2}, m_{3}\right)} s_{m_{1}+1, m_{2}, m_{3}}\right. \\
& \left.-e^{-\frac{1}{2} i \Gamma_{1}\left(m_{1}-1, m_{2}, m_{3}\right)} s_{m_{1}-1, m_{2}, m_{3}}\right) \\
& \sim \frac{1}{2} i e^{-\frac{1}{2} i m_{1} \Gamma_{1}\left(m_{I}\right)}\left(\tilde{s}_{m_{1}+1, m_{2}, m_{3}}-\tilde{s}_{m_{1}-1, m_{2}, m_{3}}\right)
\end{aligned}
$$

where

$$
\tilde{s}:=\exp \left(i \hat{p}^{I} \hat{\Gamma}_{I} / \gamma \ell_{\mathrm{P}}^{2}\right) s
$$

i.e.,

$$
\tilde{s}_{m_{1}, m_{2}, m_{3}}=\exp \left(\frac{1}{2} i \sum_{I} m_{I} \Gamma_{I}\left(m_{J}\right)\right) s_{m_{1}, m_{2}, m_{3}} .
$$

For large $m_{I}$, therefore, the operators (29) do act as basic difference operators on the wave function $\tilde{s}$ up to phase factors, and analogously operators $\widehat{\cos }\left(\frac{1}{2}\left(c_{I}-\Gamma_{I}\right)\right)$ act as basic mean operators.

Using the wave function $\tilde{s}$ instead of $s$, the constraint operator (26) contains, besides diagonal (potential) terms, a sum of products of a basic difference operator and a basic mean operator for two of the three discrete parameters $m_{I}$. The resulting difference equation for $\tilde{s}_{m_{1}, m_{2}, m_{3}}$ will thus be of the same form as the difference equation of the Bianchi-I model. Consequently, the quantization (26) can be expected to yield a locally stable evolution equation as in the case of Bianchi-I. We will discuss this in detail in the following subsections.

\section{A. Discrete evolution equation}

We now return to the constraint operator (26) and obtain the difference equation in detail. From the definitions it follows that,

$$
\begin{aligned}
\hat{h}_{I}(A, \Gamma)= & \frac{e^{\frac{i}{2} c_{I}} e^{-\frac{i}{2} \hat{\Gamma}_{I}}+e^{-\frac{i}{2} c_{I}} e^{\frac{i}{2} \hat{\Gamma}_{I}}}{2}+2 \Lambda_{I} \frac{e^{\frac{i}{2} c_{I}} e^{-\frac{i}{2} \hat{\Gamma}_{I}}-e^{-\frac{i}{2} c_{I}} e^{\frac{i}{2} \hat{\Gamma}_{I}}}{2 i} \\
= & : \hat{C}_{I}-2 i \Lambda_{I} \hat{S}_{I} \\
\left(\hat{h}_{I}(A, \Gamma)\right)^{-1}= & \frac{e^{\frac{i}{2} \hat{\Gamma}_{I}} e^{-\frac{i}{2} c_{I}}+e^{-\frac{i}{2} \hat{\Gamma}_{I}} e^{\frac{i}{2} c_{I}}}{2}+2 \Lambda_{I} \frac{e^{\frac{i}{2} \hat{\Gamma}_{I}} e^{-\frac{i}{2} c_{I}}-e^{-\frac{i}{2} \hat{\Gamma}_{I}} e^{\frac{i}{2} c_{I}}}{2 i} \\
\hat{h}_{K}(A)\left[\hat{h}_{K}^{-1}(A), \hat{V}\right]= & \left\{\hat{C}-\cos \left(\frac{1}{2} c_{K}^{\dagger}\right) \hat{V} \cos \left(\frac{1}{2} c_{K}\right)-\sin \left(\frac{1}{2} c_{K}\right) \hat{V} \sin \left(\frac{1}{2} c_{K}\right)\right\} \\
& -2 \Lambda_{K}\left\{\sin \left(\frac{1}{2} c_{K}\right) \hat{V} \cos \left(\frac{1}{2} c_{K}\right)-\cos \left(\frac{1}{2} c_{K}\right) \hat{V} \sin \left(\frac{1}{2} c_{K}\right)\right\}
\end{aligned}
$$


Note that the connection components $c_{I}$ do not have a 'hat' on them as these do not exist as operators. Their exponentials however are well defined, multiplicative operators. For notational convenience these 'hats' have been suppressed. The 'sin', $\hat{S}$, has been defined without the factor of $i$ also for future notational convenience.

Then the trace in (26) gives (suppressing the hats on the operators):

$$
\begin{aligned}
& 2 \gamma^{-2}\left\{C_{I} C_{J} C_{I}^{\dagger} C_{J}^{\dagger}+\right.\left.S_{I} C_{J} S_{I}^{\dagger} C_{J}^{\dagger}+C_{I} S_{J} C_{I}^{\dagger} S_{J}^{\dagger}-S_{I} S_{J} S_{I}^{\dagger} S_{J}^{\dagger}\right\} \\
& \times\left\{\hat{V}-\cos \left(\frac{1}{2} c_{K}\right) \hat{V} \cos \left(\frac{1}{2} c_{K}\right)-\sin \left(\frac{1}{2} c_{K}\right) \hat{V} \sin \left(\frac{1}{2} c_{K}\right)\right\} \\
&-2 \gamma^{-2} \epsilon_{I J K}\left\{S_{I} S_{J} C_{I}^{\dagger} C_{J}^{\dagger}-C_{I} S_{J} S_{I}^{\dagger} C_{J}^{\dagger}+S_{I} C_{J} C_{I}^{\dagger} S_{J}^{\dagger}+C_{I} C_{J} S_{I}^{\dagger} S_{J}^{\dagger}\right\} \\
& \times\left\{\sin \left(\frac{1}{2} c_{K}\right) \hat{V} \cos \left(\frac{1}{2} c_{K}\right)-\cos \left(\frac{1}{2} c_{K}\right) \hat{V} \sin \left(\frac{1}{2} c_{K}\right)\right\} \\
&-\epsilon_{I J K}\left\{\Gamma_{I} \Gamma_{J}-n^{K} \Gamma_{K}\right\}\left\{\sin \left(\frac{1}{2} c_{K}\right) \hat{V} \cos \left(\frac{1}{2} c_{K}\right)-\cos \left(\frac{1}{2} c_{K}\right) \hat{V} \sin \left(\frac{1}{2} c_{K}\right)\right\}
\end{aligned}
$$

Doing the sum over $I J K$ finally yields,

$$
\begin{aligned}
\left(\frac{\kappa \gamma^{3} \ell_{p}^{2}}{8 i}\right) \hat{H}= & \left\{C_{1} C_{2} C_{1}^{\dagger} C_{2}^{\dagger}+S_{1} C_{2} S_{1}^{\dagger} C_{2}^{\dagger}+C_{1} S_{2} C_{1}^{\dagger} S_{2}^{\dagger}-S_{1} S_{2} S_{1}^{\dagger} S_{2}^{\dagger}\right. \\
& \left.-C_{2} C_{1} C_{2}^{\dagger} C_{1}^{\dagger}-S_{2} C_{1} S_{2}^{\dagger} C_{1}^{\dagger}-C_{2} S_{1} C_{2}^{\dagger} S_{1}^{\dagger}+S_{2} S_{1} S_{2}^{\dagger} S_{1}^{\dagger}\right\} \\
& \times\left\{\hat{V}-\cos \left(\frac{1}{2} c_{3}\right) \hat{V} \cos \left(\frac{1}{2} c_{3}\right)-\sin \left(\frac{1}{2} c_{3}\right) \hat{V} \sin \left(\frac{1}{2} c_{3}\right)\right\} \\
& -\left\{S_{1} S_{2} C_{1}^{\dagger} C_{2}^{\dagger}-C_{1} S_{2} S_{1}^{\dagger} C_{2}^{\dagger}+S_{1} C_{2} C_{1}^{\dagger} S_{2}^{\dagger}+C_{1} C_{2} S_{1}^{\dagger} S_{2}^{\dagger}\right. \\
& \left.+S_{2} S_{1} C_{2}^{\dagger} C_{1}^{\dagger}-C_{2} S_{1} S_{2}^{\dagger} C_{1}^{\dagger}+S_{2} C_{1} C_{2}^{\dagger} S_{1}^{\dagger}+C_{2} C_{1} S_{2}^{\dagger} S_{1}^{\dagger}\right\} \\
& \times\left\{\sin \left(\frac{1}{2} c_{3}\right) \hat{V} \cos \left(\frac{1}{2} c_{3}\right)-\cos \left(\frac{1}{2} c_{3}\right) \hat{V} \sin \left(\frac{1}{2} c_{3}\right)\right\} \\
& -\gamma^{2}\left\{\Gamma_{1} \Gamma_{3}-n^{3} \Gamma_{3}\right\}\left\{\sin \left(\frac{1}{2} c_{3}\right) \hat{V} \cos \left(\frac{1}{2} c_{3}\right)-\cos \left(\frac{1}{2} c_{3}\right) \hat{V} \sin \left(\frac{1}{2} c_{3}\right)\right\} \\
& +\operatorname{cyclic}
\end{aligned}
$$

The terms containing $\hat{V}$ and $\hat{\Gamma}_{I}$ are both diagonal when acting on the basis vectors $\left|m_{1}, m_{2}, m_{3}\right\rangle$. They give:

$$
\begin{aligned}
\hat{\Gamma}_{I}\left|m_{1}, m_{2}, m_{3}\right\rangle & =\Gamma_{I}\left(m_{1}, m_{2}, m_{3}\right)\left|m_{1}, m_{2}, m_{3}\right\rangle \quad \text { where, } \\
\Gamma_{I}\left(m_{1}, m_{2}, m_{3}\right) & :=\frac{1}{2} \mathcal{N}_{j}^{-2}\left(m_{J} n^{K} f_{j}\left(m_{K}\right)+m_{K} n^{J} f_{j}\left(m_{J}\right)-m_{J} m_{K} n^{I} \mathcal{N}_{j}^{-2} f_{j}^{2}\left(m_{I}\right)\right)
\end{aligned}
$$

using the notation of (24).

Next,

$$
\begin{aligned}
& \left(\hat{V}-\cos \left(\frac{1}{2} c_{3}\right) \hat{V} \cos \left(\frac{1}{2} c_{3}\right)-\sin \left(\frac{1}{2} c_{3}\right) \hat{V} \sin \left(\frac{1}{2} c_{3}\right)\right)\left|m_{1}, m_{2}, m_{3}\right\rangle= \\
& \quad\left(V\left(m_{1}, m_{2}, m_{3}\right)-\frac{1}{2} V\left(m_{1}, m_{2}, m_{3}+1\right)-\frac{1}{2} V\left(m_{1}, m_{2}, m_{3}-1\right)\right)\left|m_{1}, m_{2}, m_{3}\right\rangle
\end{aligned}
$$




$$
\begin{aligned}
\left(\sin \left(\frac{1}{2} c_{3}\right) \hat{V} \cos \left(\frac{1}{2} c_{3}\right)-\cos \left(\frac{1}{2} c_{3}\right) \hat{V} \sin \left(\frac{1}{2} c_{3}\right)\right)\left|m_{1}, m_{2}, m_{3}\right\rangle= \\
\frac{i}{2}\left(V\left(m_{1}, m_{2}, m_{3}+1\right)-V\left(m_{1}, m_{2}, m_{3}-1\right)\right)\left|m_{1}, m_{2}, m_{3}\right\rangle
\end{aligned}
$$

The action of the $\hat{C}_{I}, \hat{S}_{I}, \hat{C}_{I}^{\dagger}, \hat{S}_{I}^{\dagger}$ on the basis states can be expressed as:

$$
\begin{aligned}
\hat{C}_{1}\left|m_{1}, m_{2}, m_{3}\right\rangle & =\frac{1}{2} \sum_{\epsilon_{1}= \pm 1} e^{-\frac{i}{2} \epsilon_{1} \Gamma_{1}\left(m_{1}, m_{2}, m_{3}\right)}\left|m_{1}+\epsilon_{1}, m_{2}, m_{3}\right\rangle \\
\hat{S}_{1}\left|m_{1}, m_{2}, m_{3}\right\rangle & =\frac{1}{2} \sum_{\epsilon_{1}= \pm 1} \epsilon_{1} e^{-\frac{i}{2} \epsilon_{1} \Gamma_{1}\left(m_{1}, m_{2}, m_{3}\right)}\left|m_{1}+\epsilon_{1}, m_{2}, m_{3}\right\rangle \\
\hat{C}_{1}^{\dagger}\left|m_{1}, m_{2}, m_{3}\right\rangle & =\frac{1}{2} \sum_{\epsilon_{1}^{\prime}= \pm 1} e^{-\frac{i}{2} \epsilon_{1}^{\prime} \Gamma_{1}\left(m_{1}+\epsilon_{1}^{\prime}, m_{2}, m_{3}\right)}\left|m_{1}+\epsilon_{1}^{\prime}, m_{2}, m_{3}\right\rangle \\
\hat{S}_{1}^{\dagger}\left|m_{1}, m_{2}, m_{3}\right\rangle & =-\frac{1}{2} \sum_{\epsilon_{1}^{\prime}= \pm 1} \epsilon_{1}^{\prime} e^{-\frac{i}{2} \epsilon_{1}^{\prime} \Gamma_{1}\left(m_{1}+\epsilon_{1}^{\prime}, m_{2}, m_{3}\right)}\left|m_{1}+\epsilon_{1}^{\prime}, m_{2}, m_{3}\right\rangle
\end{aligned}
$$

In each group of four terms, the phase factor will be the same since each consists of two $\hat{C}, \hat{S}$ type of terms and two of $\hat{C}^{\dagger}, \hat{S}^{\dagger}$ types of terms. We will just have different factors of the $\epsilon_{1}, \epsilon_{1}^{\prime}$. Thus, for example,

$$
\begin{aligned}
& \left\{C_{1} C_{2} C_{1}^{\dagger} C_{2}^{\dagger}+S_{1} C_{2} S_{1}^{\dagger} C_{2}^{\dagger}+C_{1} S_{2} C_{1}^{\dagger} S_{2}^{\dagger}-S_{1} S_{2} S_{1}^{\dagger} S_{2}^{\dagger}\right\}\left|m_{1}, m_{2}, m_{3}\right\rangle= \\
& \frac{1}{16} \sum_{\epsilon_{1}, \epsilon_{2}, \epsilon_{1}^{\prime}, \epsilon_{2}^{\prime}} e^{-\frac{i}{2}\left\{\left(\epsilon_{1}+\epsilon_{1}^{\prime}\right) \Gamma_{1}\left(m_{1}+\epsilon_{1}^{\prime}, m_{2}+\epsilon_{2}^{\prime}, m_{3}\right)+\epsilon_{2} \Gamma_{2}\left(m_{1}+\epsilon_{1}^{\prime}, m_{2}+\epsilon_{2}^{\prime}, m_{3}\right)+\epsilon_{2}^{\prime} \Gamma_{2}\left(m_{1}, m_{2}+\epsilon_{2}^{\prime}, m_{3}\right)\right\}} \\
& \times\left(1-\epsilon_{1} \epsilon_{1}^{\prime}-\epsilon_{2} \epsilon_{2}^{\prime}-\epsilon_{1} \epsilon_{1}^{\prime} \epsilon_{2} \epsilon_{2}^{\prime}\right) \quad\left|m_{1}+\epsilon_{1}+\epsilon_{1}^{\prime}, m_{2}+\epsilon_{2}+\epsilon_{2}^{\prime}, m_{3}\right\rangle, \\
& \left\{C_{2} C_{1} C_{2}^{\dagger} C_{1}^{\dagger}+S_{2} C_{1} S_{2}^{\dagger} C_{1}^{\dagger}+C_{2} S_{1} C_{2}^{\dagger} S_{1}^{\dagger}-S_{2} S_{1} S_{2}^{\dagger} S_{1}^{\dagger}\right\}\left|m_{1}, m_{2}, m_{3}\right\rangle= \\
& \frac{1}{16} \sum_{\epsilon_{1}, \epsilon_{2}, \epsilon_{1}^{\prime}, \epsilon_{2}^{\prime}} e^{-\frac{i}{2}\left\{\left(\epsilon_{2}+\epsilon_{2}^{\prime}\right) \Gamma_{2}\left(m_{1}+\epsilon_{1}^{\prime}, m_{2}+\epsilon_{2}^{\prime}, m_{3}\right)+\epsilon_{1} \Gamma_{1}\left(m_{1}+\epsilon_{1}^{\prime}, m_{2}+\epsilon_{2}^{\prime}, m_{3}\right)+\epsilon_{1}^{\prime} \Gamma_{1}\left(m_{1}+\epsilon_{1}^{\prime}, m_{2}, m_{3}\right)\right\}} \\
& \times\left(1-\epsilon_{1} \epsilon_{1}^{\prime}-\epsilon_{2} \epsilon_{2}^{\prime}-\epsilon_{1} \epsilon_{1}^{\prime} \epsilon_{2} \epsilon_{2}^{\prime}\right) \quad\left|m_{1}+\epsilon_{1}+\epsilon_{1}^{\prime}, m_{2}+\epsilon_{2}+\epsilon_{2}^{\prime}, m_{3}\right\rangle, \\
& \left\{S_{1} S_{2} C_{1}^{\dagger} C_{2}^{\dagger}-C_{1} S_{2} S_{1}^{\dagger} C_{2}^{\dagger}+S_{1} C_{2} C_{1}^{\dagger} S_{2}^{\dagger}+C_{1} C_{2} S_{1}^{\dagger} S_{2}^{\dagger}\right\}\left|m_{1}, m_{2}, m_{3}\right\rangle= \\
& \frac{1}{16} \sum_{\epsilon_{1}, \epsilon_{2}, \epsilon_{1}^{\prime}, \epsilon_{2}^{\prime}} e^{-\frac{i}{2}\left\{\left(\epsilon_{1}+\epsilon_{1}^{\prime}\right) \Gamma_{1}\left(m_{1}+\epsilon_{1}^{\prime}, m_{2}+\epsilon_{2}^{\prime}, m_{3}\right)+\epsilon_{2} \Gamma_{2}\left(m_{1}+\epsilon_{1}^{\prime}, m_{2}+\epsilon_{2}^{\prime}, m_{3}\right)+\epsilon_{2}^{\prime} \Gamma_{2}\left(m_{1}, m_{2}+\epsilon_{2}^{\prime}, m_{3}\right)\right\}} \\
& \times\left(\epsilon_{1} \epsilon_{2}+\epsilon_{1}^{\prime} \epsilon_{2}-\epsilon_{1} \epsilon_{2}^{\prime}+\epsilon_{1}^{\prime} \epsilon_{2}^{\prime}\right)\left|m_{1}+\epsilon_{1}+\epsilon_{1}^{\prime}, m_{2}+\epsilon_{2}+\epsilon_{2}^{\prime}, m_{3}\right\rangle
\end{aligned}
$$

and

$$
\left\{S_{2} S_{1} C_{2}^{\dagger} C_{1}^{\dagger}-C_{2} S_{1} S_{2}^{\dagger} C_{1}^{\dagger}+S_{2} C_{1} C_{2}^{\dagger} S_{1}^{\dagger}+C_{2} C_{1} S_{2}^{\dagger} S_{1}^{\dagger}\right\}\left|m_{1}, m_{2}, m_{3}\right\rangle=
$$




$$
\begin{aligned}
& \frac{1}{16} \sum_{\epsilon_{1}, \epsilon_{2}, \epsilon_{1}^{\prime}, \epsilon_{2}^{\prime}} e^{-\frac{i}{2}\left\{\left(\epsilon_{2}+\epsilon_{2}^{\prime}\right) \Gamma_{2}\left(m_{1}+\epsilon_{1}^{\prime}, m_{2}+\epsilon_{2}^{\prime}, m_{3}\right)+\epsilon_{1} \Gamma_{1}\left(m_{1}+\epsilon_{1}^{\prime}, m_{2}+\epsilon_{2}^{\prime}, m_{3}\right)+\epsilon_{1}^{\prime} \Gamma_{1}\left(m_{1}+\epsilon_{1}^{\prime}, m_{2}, m_{3}\right)\right\}} \\
& \times\left(\epsilon_{1} \epsilon_{2}-\epsilon_{1}^{\prime} \epsilon_{2}+\epsilon_{1} \epsilon_{2}^{\prime}+\epsilon_{1}^{\prime} \epsilon_{2}^{\prime}\right) \quad\left|m_{1}+\epsilon_{1}+\epsilon_{1}^{\prime}, m_{2}+\epsilon_{2}+\epsilon_{2}^{\prime}, m_{3}\right\rangle .
\end{aligned}
$$

It is convenient to define different basis vectors at this stage, namely,

$$
\left|m_{1}, m_{2}, m_{3}\right\rangle:=e^{\frac{1}{2} i \sum_{I} m_{I} \Gamma_{I}\left(m_{1}, m_{2}, m_{3}\right)}\left|m_{1} \widetilde{m_{2},} m_{3}\right\rangle
$$

corresponding to the transformation (30). This introduces additional phases which will turn out to be useful in our discussion of the continuum approximation. The transformation to the triad representation via,

$$
|s\rangle:=\sum_{m_{1}, m_{2}, m_{3}} \tilde{s}_{m_{1}, m_{2}, m_{3}}\left|\mathrm{~m}_{1} \widetilde{m_{2}, m_{3}}\right\rangle,
$$

leads to an equation for the $\tilde{s}_{m_{1}, m_{2}, m_{3}}$ :

$$
\begin{gathered}
0=\sum_{\epsilon_{1}, \epsilon_{2}, \epsilon_{1}^{\prime}, \epsilon_{2}^{\prime}} \gamma^{-2} A_{12}\left(m_{1}, m_{2}, m_{3} ; \epsilon_{1}, \epsilon_{2}, \epsilon_{1}^{\prime}, \epsilon_{2}^{\prime}\right) \tilde{s}_{m_{1}-\epsilon_{1}-\epsilon_{1}^{\prime}, m_{2}-\epsilon_{2}-\epsilon_{2}^{\prime}, m_{3}} \\
+\sum_{\epsilon_{2}, \epsilon_{3}, \epsilon_{2}^{\prime}, \epsilon_{3}^{\prime}} \gamma^{-2} A_{23}\left(m_{1}, m_{2}, m_{3} ; \epsilon_{2}, \epsilon_{3}, \epsilon_{2}^{\prime}, \epsilon_{3}^{\prime}\right) \tilde{s}_{m_{1}, m_{2}-\epsilon_{2}-\epsilon_{2}^{\prime}, m_{3}-\epsilon_{3}-\epsilon_{3}^{\prime}} \\
+\sum_{\epsilon_{3}, \epsilon_{1}, \epsilon_{3}^{\prime}, \epsilon_{1}^{\prime}} \gamma^{-2} A_{31}\left(m_{1}, m_{2}, m_{3} ; \epsilon_{3}, \epsilon_{1}, \epsilon_{3}^{\prime}, \epsilon_{1}^{\prime}\right) \tilde{s}_{m_{1}-\epsilon_{1}-\epsilon_{1}^{\prime}, m_{2}, m_{3}-\epsilon_{3}-\epsilon_{3}^{\prime}} \\
\quad+B\left(m_{1}, m_{2}, m_{3}\right) \tilde{s}_{m_{1}, m_{2}, m_{3}}
\end{gathered}
$$

where the coefficients are rather complicated expressions given by:

$$
\begin{aligned}
v_{12}^{ \pm}\left(m_{1}, m_{2}, m_{3} ; \epsilon_{1}, \epsilon_{2}, \epsilon_{1}^{\prime}, \epsilon_{2}^{\prime}\right):= & V\left(m_{1}-\epsilon_{1}-\epsilon_{1}^{\prime}, m_{2}-\epsilon_{2}-\epsilon_{2}^{\prime}, m_{3}+1\right) \\
\pm & V\left(m_{1}-\epsilon_{1}-\epsilon_{1}^{\prime}, m_{2}-\epsilon_{2}-\epsilon_{2}^{\prime}, m_{3}-1\right), \\
u_{12}\left(m_{1}, m_{2}, m_{3} ; \epsilon_{1}, \epsilon_{2}, \epsilon_{1}^{\prime}, \epsilon_{2}^{\prime}\right):=V\left(m_{1}-\epsilon_{1}-\epsilon_{1}^{\prime}, m_{2}-\epsilon_{2}-\epsilon_{2}^{\prime}, m_{3}\right) & -\frac{1}{2} v_{12}^{+}\left(m_{1}, m_{2}, m_{3} ; \epsilon_{1}, \epsilon_{2}, \epsilon_{1}^{\prime}, \epsilon_{2}^{\prime}\right), \\
\phi_{12,1}\left(m_{1}, m_{2}, m_{3} ; \epsilon_{1}, \epsilon_{2}, \epsilon_{1}^{\prime}, \epsilon_{2}^{\prime}\right):= & -\sum_{I} m_{I} \Gamma_{I}\left(m_{1}, m_{2}, m_{3}\right) \\
& +\left(\epsilon_{1}+\epsilon_{1}^{\prime}\right) \Gamma_{1}\left(m_{1}-\epsilon_{1}, m_{2}-\epsilon_{2}, m_{3}\right) \\
& +\left(\epsilon_{2}+\epsilon_{2}^{\prime}\right) \Gamma_{2}\left(m_{1}-\epsilon_{1}, m_{2}-\epsilon_{2}, m_{3}\right) \\
& +\epsilon_{1}^{\prime}\left\{\Gamma_{1}\left(m_{1}-\epsilon_{1}, m_{2}-\epsilon_{2}-\epsilon_{2}^{\prime}, m_{3}\right)\right. \\
& \left.-\Gamma_{1}\left(m_{1}-\epsilon_{1}, m_{2}-\epsilon_{2}, m_{3}\right)\right\}
\end{aligned}
$$


and

$$
\begin{aligned}
\phi_{12,2}\left(m_{1}, m_{2}, m_{3} ; \epsilon_{1}, \epsilon_{2}, \epsilon_{1}^{\prime}, \epsilon_{2}^{\prime}\right):= & -\sum_{I} m_{I} \Gamma_{I}\left(m_{1}, m_{2}, m_{3}\right) \\
& +\left(\epsilon_{1}+\epsilon_{1}^{\prime}\right) \Gamma_{1}\left(m_{1}-\epsilon_{1}, m_{2}-\epsilon_{2}, m_{3}\right) \\
& +\left(\epsilon_{2}+\epsilon_{2}^{\prime}\right) \Gamma_{2}\left(m_{1}-\epsilon_{1}, m_{2}-\epsilon_{2}, m_{3}\right) \\
& +\epsilon_{2}^{\prime}\left\{\Gamma_{2}\left(m_{1}-\epsilon_{1}-\epsilon_{1}^{\prime}, m_{2}-\epsilon_{2}, m_{3}\right)\right. \\
& \left.-\Gamma_{2}\left(m_{1}-\epsilon_{1}, m_{2}-\epsilon_{2}, m_{3}\right)\right\}
\end{aligned}
$$

With obvious abbreviations, e.g., $\left(m_{1}, m_{2}, m_{3}\right) \equiv \vec{m},\left(\epsilon_{1}, \epsilon_{2}, \epsilon_{1}^{\prime}, \epsilon_{2}^{\prime}\right) \equiv \vec{\epsilon}_{12}$ and $A_{12}\left(m_{1}, m_{2}, m_{3} ; \epsilon_{1}, \epsilon_{2}, \epsilon_{1}^{\prime}, \epsilon_{2}^{\prime}\right) \equiv A_{12}\left(\vec{m} ; \vec{\epsilon}_{12}\right)$ etc. , we have

$$
\begin{aligned}
A_{12}\left(\vec{m} ; \vec{\epsilon}_{12}\right):=i u_{12}\left(\vec{m} ; \vec{\epsilon}_{12}\right)\left(1-\epsilon_{1} \epsilon_{1}^{\prime}-\epsilon_{2} \epsilon_{2}^{\prime}-\epsilon_{1} \epsilon_{1}^{\prime} \epsilon_{2} \epsilon_{2}^{\prime}\right)\left(e^{-\frac{i}{2} \phi_{12,2}\left(\vec{m} ; \vec{\epsilon}_{12}\right)}-e^{-\frac{i}{2} \phi_{12,1}\left(\vec{m} ; \vec{\epsilon}_{12}\right)}\right) \\
+\frac{1}{2} v_{12}^{-}\left(\vec{m} ; \vec{\epsilon}_{12}\right)\left\{e^{-\frac{i}{2} \phi_{12,2}\left(\vec{m} ; \vec{\epsilon}_{12}\right)}\left(\epsilon_{1} \epsilon_{2}+\epsilon_{1}^{\prime} \epsilon_{2}-\epsilon_{1} \epsilon_{2}^{\prime}+\epsilon_{1}^{\prime} \epsilon_{2}^{\prime}\right)\right. \\
\left.+e^{-\frac{i}{2} \phi_{12,1}\left(\vec{m} ; \vec{\epsilon}_{12}\right)}\left(\epsilon_{1} \epsilon_{2}-\epsilon_{1}^{\prime} \epsilon_{2}+\epsilon_{1} \epsilon_{2}^{\prime}+\epsilon_{1}^{\prime} \epsilon_{2}^{\prime}\right)\right\}
\end{aligned}
$$

and

$$
\begin{aligned}
B(\vec{m}):= & 8\left(e^{\frac{i}{2} \sum_{I} m_{I} \Gamma_{I}(\vec{m})}\right) \\
& \times\left[v_{12}^{-}(\vec{m} ; \overrightarrow{0})\left\{\Gamma_{1}(\vec{m}) \Gamma_{2}(\vec{m})-n^{3} \Gamma_{3}(\vec{m})\right\}\right. \\
& v_{23}^{-}(\vec{m} ; \overrightarrow{0})\left\{\Gamma_{2}(\vec{m}) \Gamma_{3}(\vec{m})-n^{1} \Gamma_{1}(\vec{m})\right\} \\
& \left.v_{31}^{-}(\vec{m} ; \overrightarrow{0})\left\{\Gamma_{3}(\vec{m}) \Gamma_{1}(\vec{m})-n^{2} \Gamma_{2}(\vec{m})\right\}\right] \\
& -2 \kappa \gamma \ell_{p}^{2}\left(e^{\frac{i}{2} \sum_{I} m_{I} \Gamma_{I}(\vec{m})}\right) \hat{H}_{m_{1}, m_{2}, m_{3}}^{\text {matter }}
\end{aligned}
$$

The matter Hamiltonian acting on the matter field dependence of the wave function has also been incorporated in the coefficient $B(\vec{m})$.

Noting that the coefficients $u_{12}, A_{12}, B$ involve $v_{12}^{ \pm}, V$ and that the volume eigenvalues have a separable product structure, we can absorb the volume factors in the wave functions as follows. Define,

$$
d^{ \pm}(m):=\left\{\begin{array}{cc}
\sqrt{1+m^{-1}} \pm \sqrt{1-m^{-1}} & \text { if } m \neq 0 \\
0 & \text { if } m=0
\end{array}\right.
$$

Then,

$$
\begin{aligned}
& v_{12}^{ \pm}(\vec{m} ; \vec{\epsilon})=V\left(m_{1}-\epsilon_{1}-\epsilon_{1}^{\prime}, m_{2}-\epsilon_{2}-\epsilon_{2}^{\prime}, m_{3}\right) d^{ \pm}\left(m_{3}\right) \\
& u_{12}(\vec{m} ; \vec{\epsilon})=V\left(m_{1}-\epsilon_{1}-\epsilon_{1}^{\prime}, m_{2}-\epsilon_{2}-\epsilon_{2}^{\prime}, m_{3}\right)\left\{1-\frac{d^{+}\left(m_{3}\right)}{2}\right\}
\end{aligned}
$$


Now defining $\tilde{t}_{m_{1}, m_{2}, m_{3}}:=V(\vec{m}) \tilde{s}_{m_{1}, m_{2}, m_{3}}$ and $\hat{\rho}_{m_{1}, m_{2}, m_{3}}^{\text {matter }}:=\hat{H}_{m_{1}, m_{2}, m_{3}}^{\text {matter }} / V(\vec{m})$ for $V\left(m_{1}, m_{2}, m_{3}\right) \neq 0$, one sees that all volume eigenvalue factors are absorbed in the wave function. This preserves the gauge invariance condition on the $\tilde{S}$ but now in addition, due to the explicit volume eigenvalues, we also have that $\tilde{t}_{m_{1}, m_{2}, m_{3}}=0$ if any of the $m_{i}$ 's equal zero.

For Bianchi I, the spin connection is zero. The phases $\phi_{12,1}, \phi_{12,2}, \ldots$ vanish. The coefficient $B$ reduces to the matter term only, while $A_{12}$ reduces to :

$$
A_{12}\left(\vec{m} ; \vec{\epsilon}_{12}\right)=d^{-}\left(m_{3}\right)\left(\epsilon_{1} \epsilon_{2}+\epsilon_{1}^{\prime} \epsilon_{2}^{\prime}\right)
$$

Equation (50) then becomes,

$$
\begin{aligned}
& d^{-}\left(m_{1}\right)\left(\tilde{t}_{m_{1}, m_{2}+2, m_{3}+2}+\tilde{t}_{m_{1}, m_{2}-2, m_{3}-2}-\tilde{t}_{m_{1}, m_{2}+2, m_{3}-2}-\tilde{t}_{m_{1}, m_{2}-2, m_{3}+2}\right) \\
+ & d^{-}\left(m_{2}\right)\left(\tilde{t}_{m_{1}+2, m_{2}, m_{3}+2}+\tilde{t}_{m_{1}-2, m_{2}, m_{3}-2}-\tilde{t}_{m_{1}-2, m_{2}, m_{3}+2}-\tilde{t}_{m_{1}+2, m_{2}, m_{3}-2}\right) \\
+ & d^{-}\left(m_{3}\right)\left(\tilde{t}_{m_{1}+2, m_{2}+2, m_{3}}+\tilde{t}_{m_{1}-2, m_{2}-2, m_{3}}-\tilde{t}_{m_{1}+2, m_{2}-2, m_{3}}-\tilde{t}_{m_{1}-2, m_{2}+2, m_{3}}\right) \\
= & -2 \kappa \gamma^{3} \ell_{p}^{2} \hat{\rho}_{m_{1}, m_{2}, m_{3}}^{\text {matter }} \tilde{t}_{m_{1}, m_{2}, m_{3}}
\end{aligned}
$$

which matches with the equation derived in [6].

\section{B. Continuum approximation}

We will first derive a simplified difference equation which approximates the exact one for large $m_{I}$. Then using an interpolating function, we will show that the approximate difference equation gives a differential equation for the interpolating function, which matches with the Wheeler-DeWitt equation at the level of leading terms. This will verify that the continuum approximation is admissible.

Consider first $m_{I} \gg 1$. Then, for $k \ll m, f_{j}(m+k) \rightarrow \frac{\mathcal{N}_{j}^{2}}{m}$ which is independent of $k$ to the leading order. This implies that

$$
\Gamma_{I} \sim \frac{1}{2}\left\{n^{K} \frac{m_{J}}{m_{K}}+n^{J} \frac{m_{K}}{m_{J}}-n^{I} \frac{m_{J} m_{K}}{m_{I}^{2}}\right\} .
$$

The phases $\phi_{12,1}, \phi_{12,2}$ etc. all become equal to the common phase appearing in the coefficient $B$ and cancel out. The coefficients $A_{I J}$ become exactly the same as in Bianchi-I with $d^{-}\left(m_{I}\right) \approx m_{I}^{-1}$ and the equation becomes,

$$
-2 \kappa \gamma \ell_{p}^{2} \hat{\rho}_{m_{1}, m_{2}, m_{3}}^{\text {matter }} \tilde{t}_{m_{1}, m_{2}, m_{3}}=\gamma^{-2} m_{3}^{-1} \sum_{\epsilon_{1}, \epsilon_{2}, \epsilon_{1}^{\prime}, \epsilon_{2}^{\prime}}\left(\epsilon_{1} \epsilon_{2}+\epsilon_{1}^{\prime} \epsilon_{2}^{\prime}\right) \tilde{t}_{m_{1}-\epsilon_{1}-\epsilon_{1}^{\prime}, m_{2}-\epsilon_{2}-\epsilon_{2}^{\prime}, m_{3}}
$$




$$
+8 m_{3}^{-1}\left(\Gamma_{1} \Gamma_{2}-n^{3} \Gamma_{3}\right) \tilde{t}_{m_{1}, m_{2}, m_{3}}+\text { cyclic }
$$

To arrive at a differential equation, we look for a function $\tilde{T}\left(p^{1}, p^{2}, p^{3}\right)$ such that $\tilde{t}_{m_{1}, m_{2}, m_{3}}:=\tilde{T}\left(p^{1}\left(m_{1}\right), p^{2}\left(m_{2}\right), p^{3}\left(m_{3}\right)\right)$ satisfies the approximate difference equation. Here, $p^{I}\left(m_{I}\right):=\frac{1}{2} \gamma \ell_{p}^{2} m_{I}$ and thus $\delta p^{I}=\frac{1}{2} \gamma \ell_{p}^{2}$ is the change induced when the $m_{I}$ change by 1 . In particular, this implies:

$$
\begin{aligned}
\tilde{t}_{m_{1}+k_{1}, m_{2}+k_{2}, m_{3}+k_{3}} & :=\tilde{T}\left(p^{I}\left(m_{I}+k_{I}\right)\right)=\tilde{T}\left(p^{I}\left(m_{I}\right)+k_{I} \delta p^{I}\right) \\
\approx \tilde{T} & \left(p^{I}\left(m_{I}\right)\right)+\sum_{I} k_{I} \delta p^{I} \frac{\partial \tilde{T}}{\partial p^{I}}+\frac{1}{2} \sum_{I J} k_{I} k_{J} \delta p^{I} \delta p^{J} \frac{\partial^{2} \tilde{T}}{\partial p^{I} \partial p^{J}} \\
& \quad+\quad \ldots
\end{aligned}
$$

It follows that terms of order $\left(\delta p^{I}\right)^{0},\left(\delta p^{I}\right)^{1}$ cancel out leaving a second order differential expression to the leading order. We also have the pre-factors $\gamma^{-2} m_{I}^{-1}$ which can be eliminated using $m_{I}=\frac{2 p^{I}}{\ell_{p}^{2}} \gamma^{-1}$. The spin connection then becomes just the classical expression without any factors of $\gamma \ell_{p}^{2}$. All factors of $\gamma$ cancel out. Multiplying by $\frac{1}{8} p^{1} p^{2} p^{3}$ the final equation becomes:

$$
\begin{aligned}
& {\left[\frac{\ell_{p}^{4}}{4} p^{1} p^{2} \frac{\partial^{2} \tilde{T}\left(p^{1}, p^{2}, p^{3}\right)}{\partial p^{1} \partial p^{2}}+p^{1} p^{2}\left(\Gamma_{1} \Gamma_{2}-n^{3} \Gamma_{3}\right) \tilde{T}\left(p^{1}, p^{2}, p^{3}\right)\right]+\text { cyclic }} \\
& =-\frac{1}{2} \kappa p^{1} p^{2} p^{3} \hat{\rho}^{\text {matter }}\left(p^{1}, p^{2}, p^{3}\right) \tilde{T}\left(p^{1}, p^{2}, p^{3}\right)
\end{aligned}
$$

We have thus verified that the constraint operator (26), admits a continuum approximation.

Note that using an interpolating function, $\tilde{T}\left(p^{1}, p^{2}, p^{3}\right)$, one can always arrive at some differential expression. That all dependence on $\gamma$ disappears from the differential equation is a non-trivial property of the locally approximated difference equation and is essential for matching with the Wheeler-DeWitt equation which knows nothing about the BarberoImmirzi parameter. Had the phases not canceled, we would have acquired terms from the Taylor expansion of the $\Gamma_{I}$, which could have produced lower order differentials with a $\gamma$ dependence left over, destroying the hope of matching with the Wheeler-DeWitt equation. That the spin connection controls the phases in a cancelable manner is thus also a nontrivial property of the constraint operator. Finally, the fact that we get a purely second order differential expressions is determined by the structure of the coefficients in the locally approximated difference equation [11]. This is directly responsible for getting the WheelerDeWitt equation in a particular factor ordering appearing in (65). 
The wave function $\tilde{T}$ has been obtained after some redefinitions. Since $V(\vec{m}) \rightarrow \sqrt{\left|p^{1} p^{2} p^{3}\right|}$ in the pre-classical limit, $\tilde{T}$ is related to $\tilde{S}$ as $\tilde{T} \sim \sqrt{\left|p^{1} p^{2} p^{3}\right|} \tilde{S}$. Thus, the Wheeler-DeWitt equation for our original pre-classical wave function $\tilde{S}$ is

$$
\begin{gathered}
{\left[\frac{\ell_{p}^{4}}{4} p^{1} p^{2} \frac{\partial^{2} \sqrt{\left|p^{1} p^{2} p^{3}\right|} \tilde{S}\left(p^{1}, p^{2}, p^{3}\right)}{\partial p^{1} \partial p^{2}}+p^{1} p^{2}\left(\Gamma_{1} \Gamma_{2}-n^{3} \Gamma_{3}\right) \sqrt{\left|p^{1} p^{2} p^{3}\right|} \tilde{S}\left(p^{1}, p^{2}, p^{3}\right)\right]+\text { cyclic }} \\
=-\frac{1}{2} \kappa\left|p^{1} p^{2} p^{3}\right|^{\frac{3}{2}} \hat{\rho}^{\text {matter }}\left(p^{1}, p^{2}, p^{3}\right) \tilde{S}\left(p^{1}, p^{2}, p^{3}\right)
\end{gathered}
$$

Note that the ordering of an isotropic sub-model is not determined uniquely by using $p^{1}=$ $p^{2}=p^{3}=: p$ because $p^{1}$ and $\partial / \partial p^{2}$, say, commute in the anisotropic model but not after using isotropy. However, there is also a unique ordering for isotropic models [8, 21] which can be derived after doing the reduction in loop quantum cosmology.

\section{Local Stability}

Our next requirement is about local stability of the evolution equation around pre-classical solutions. This is most directly formulated and illustrated in the context of an ordinary difference equation [11]. The equation we have however is a partial difference equation. Although, we do get a partial difference equation with constant coefficients in a local approximation, their solutions are not as easily analyzable in terms of roots of some polynomial. Nevertheless, the property of local stability can still be analyzed.

Since we have three $m_{I}$ 's, even if all are taken to be large, it is possible to have a subset of them to be much larger compared to the remaining ones. To begin with, we consider a neighborhood of a point where all $m_{I}$ are large and of the same order; other regions can be explored similarly. For $\gamma$ we assume the value derived from black hole entropy calculations which is of order $10^{-1}$. Since all $m_{I}$ 's are large, it follows exactly as before, that $\Gamma_{I}$ are given by the equation (62) and all phases cancel out giving the locally approximated difference equation (63). Furthermore, since we assume the $m_{I}$ to be about the same size, the $\Gamma_{I}$ are approximately equal and taking the Bianchi IX model for definiteness, $\Gamma_{I} \approx \frac{1}{2}$. We obtain

$$
\begin{aligned}
& d^{-}\left(m_{1}\right)\left(\tilde{t}_{m_{1}, m_{2}+2, m_{3}+2}+\tilde{t}_{m_{1}, m_{2}-2, m_{3}-2}-\tilde{t}_{m_{1}, m_{2}+2, m_{3}-2}-\tilde{t}_{m_{1}, m_{2}-2, m_{3}+2}\right) \\
+ & d^{-}\left(m_{2}\right)\left(\tilde{t}_{m_{1}+2, m_{2}, m_{3}+2}+\tilde{t}_{m_{1}-2, m_{2}, m_{3}-2}-\tilde{t}_{m_{1}-2, m_{2}, m_{3}+2}-\tilde{t}_{m_{1}+2, m_{2}, m_{3}-2}\right) \\
+ & d^{-}\left(m_{3}\right)\left(\tilde{t}_{m_{1}+2, m_{2}+2, m_{3}}+\tilde{t}_{m_{1}-2, m_{2}-2, m_{3}}-\tilde{t}_{m_{1}-2, m_{2}+2, m_{3}}-\tilde{t}_{m_{1}+2, m_{2}-2, m_{3}}\right) \\
= & -2 \kappa \gamma^{3} \ell_{\mathrm{P}}^{2} \hat{\rho}_{m_{1}, m_{2}, m_{3}}^{\text {matter }} \tilde{t}_{m_{1}, m_{2}, m_{3}}+2 \gamma^{2}\left(d^{-}\left(m_{1}\right)+d^{-}\left(m_{2}\right)+d^{-}\left(m_{3}\right)\right) \tilde{t}_{m_{1}, m_{2}, m_{3}} .
\end{aligned}
$$


In this equation, $m_{1}, m_{2}$ and $m_{3}$ all play the same role, but without loss of generality we can select $m_{3}$ to be the evolution parameter in terms of which we analyze local stability. To express it clearly, let us introduce the notation

$$
z_{m_{3}}^{\nu_{1}, \nu_{2}}:=\tilde{t}_{m_{1}+\nu_{1}, m_{2}+\nu_{2}, m_{3}}, \quad \nu_{1}, \nu_{2}=0, \pm 2
$$

which allows us to write the equation as

$$
\begin{aligned}
& d^{-}\left(m_{1}\right)\left(z_{m_{3}+2}^{0,2}-z_{m_{3}-2}^{0,2}\right)-d^{-}\left(m_{1}\right)\left(z_{m_{3}+2}^{0,-2}-z_{m_{3}-2}^{0,-2}\right) \\
& \quad+d^{-}\left(m_{2}\right)\left(z_{m_{3}+2}^{2,0}-z_{m_{3}-2}^{2,0}\right)-d^{-}\left(m_{2}\right)\left(z_{m_{3}+2}^{-2,0}-z_{m_{3}-2}^{-2,0}\right) \\
& =-d^{-}\left(m_{3}\right)\left(z_{m_{3}}^{2,2}+z_{m_{3}}^{-2,2}-z_{m_{3}}^{2,-2}-z_{m_{3}}^{-2,-2}\right) \\
& -2 \gamma^{2}\left(\gamma P+d^{-}\left(m_{1}\right)+d^{-}\left(m_{2}\right)+d^{-}\left(m_{3}\right)\right) z_{m_{3}}^{0,0}
\end{aligned}
$$

where

$$
P:=\kappa \ell_{\mathrm{P}}^{2} \rho^{\text {matter }}
$$

is a small constant (in the local approximation) characterizing the size of the matter contribution.

For $m_{I}$ 's of the same order $m$, the equation can be further simplified as

$$
\begin{aligned}
& \left(z_{m_{3}+2}^{0,2}-z_{m_{3}-2}^{0,2}\right)-\left(z_{m_{3}+2}^{0,-2}-z_{m_{3}-2}^{0,-2}\right)+\left(z_{m_{3}+2}^{2,0}-z_{m_{3}-2}^{2,0}\right)-\left(z_{m_{3}+2}^{-2,0}-z_{m_{3}-2}^{-2,0}\right) \\
\approx & -\left(z_{m_{3}}^{2,2}+z_{m_{3}}^{-2,2}-z_{m_{3}}^{2,-2}-z_{m_{3}}^{-2,-2}\right)-2 \gamma^{2}(3+\gamma m P) z_{m_{3}}^{0,0}
\end{aligned}
$$

We are interested in checking if perturbation of slowly varying (along all three directions) solutions, continue to remain as perturbations under the $m_{3}$-evolution. To this end, we assume that we have some fixed solution which is slowly varying. Then the first bracket on the right hand side is small while the second one is suppressed by $\gamma^{2} \sim 10^{-2}(\mathrm{mP}$ is small even for large $m$ because the energy density decreases at least as $m^{-3}$ ).

This is a linear equation among four combinations analogous to the equation of a plane in $\mathbb{R}^{4}$ passing close to the origin. It can be solved approximately by introducing three, fixed orthonormal vectors, $\overrightarrow{\mathcal{E}}^{a}, a=1,2,3$, each of which is orthogonal to the vector $(1,-1,1,-1)$ in $\mathbb{R}^{4}$. Explicitly,

$$
\left(z_{m_{3}+2}^{0,2}-z_{m_{3}-2}^{0,2}\right) \approx \sum_{a=1}^{3} Q_{a}\left(m_{3}\right)\left(\mathcal{E}^{a}\right)_{0,2}
$$




$$
\begin{aligned}
\left(z_{m_{3}+2}^{0,-2}-z_{m_{3}-2}^{0,-2}\right) & \approx \sum_{a=1}^{3} Q_{a}\left(m_{3}\right)\left(\mathcal{E}^{a}\right)_{0,-2} \\
\left(z_{m_{3}+2}^{2,0}-z_{m_{3}-2}^{2,0}\right) & \approx \sum_{a=1}^{3} Q_{a}\left(m_{3}\right)\left(\mathcal{E}^{a}\right)_{2,0} \\
\left(z_{m_{3}+2}^{-2,0}-z_{m_{3}-2}^{-2,0}\right) & \approx \sum_{a=1}^{3} Q_{a}\left(m_{3}\right)\left(\mathcal{E}^{a}\right)_{-2,0}
\end{aligned}
$$

In the above, corrections of the order $z_{m_{3}}^{\nu_{1}, \nu_{2}}-z_{m_{3}}^{\nu_{1}^{\prime}, \nu_{2}^{\prime}}$ as well as of the order $\gamma^{2}(3+\gamma m P) z_{m_{3}}^{0,0}$ are ignored and $Q_{a}\left(m_{3}\right)$ are three functions of $m_{3}$. The $Q_{a}\left(m_{3}\right)$ can be assumed to be small for $z$ to represent a solution close to a pre-classical one.

Thus we obtain ordinary, non-homogeneous difference equations with constant coefficients for the four $z_{m_{3}}^{\nu_{1}, \nu_{2}}$. All the equations have the same structure, and their solutions consist of a combination of the particular solution and linear combinations of solutions of the corresponding homogeneous equations. The difference between a perturbed solution and the fixed solution of course satisfies the homogeneous equations.

The local stability now requires that these differences do not grow exponentially. Equivalently, the characteristic roots for the homogeneous equations must have absolute value less than or equal to one.

In our case, the characteristic polynomials are the same for all four equations namely, $z^{4}-1=0$, with roots equal to $\pm 1, \pm i$. These obviously satisfy the condition of local stability.

Because of local stability, perturbations of slowly varying solutions continue to remain close to them under evolution along the $m_{3}$ direction. This also ensures self-consistency when we consider evolutions along the $m_{1}, m_{2}$ directions in a similar manner by keeping the corresponding right hand sides close to zero.

Had we not neglected the terms on the right hand side of eq. (69), we would have got an extra, common non-homogeneous term which is $1 / 2$ of the right hand side of eq. (69). Clearly, this does not affect the behavior of the differences.

To summarize, although we got a partial difference equation, we were able to implement the idea behind the local stability criterion by obtaining a system of ordinary difference equations. That these equations are non-homogeneous, does not affect the stability properties 11]. 


\section{Absence of Singularities}

We have now verified that the proposed quantization (26) has a valid continuum approximation and is locally stable around pre-classical solutions. Thus, it is possible to start with initial data at large volume which are close to a continuum solution and to follow the evolution to smaller volume, toward the classical singularity. In this regime, quantum effects of the discreteness and also ordering issues become more and more important and the full equation has to be considered without approximations. Fortunately, it is possible to see essential features of the absence of singularities quite generally in a way similar to the isotropic case [9]. Since the constraint equation is used as a recurrence relation for the wave function, the term with lowest order in the labels $m_{I}$ should have non-vanishing coefficient, except when one of the labels vanishes such that the corresponding component of the wave function, i.e. its value at singular surfaces in minisuperspace, will drop out (see [6] for details). It can readily be verified that this general behavior is true for the evolution equation of an arbitrary Bianchi class A model. The difference equation resulting from (26) is more complicated than in the isotropic [8] or Bianchi I [6] case; but since the exponentiated spin connection never vanishes and the additional potential term containing the spin connection is diagonal, only the commutator of holonomies with the volume operator determines whether or not a lowest order coefficient vanishes. The structure resulting from this term is the same as in the Bianchi I case, and thus the evolution is singularity-free in the same way.

This shows that the wave function can be extended uniquely from the region of minisuperspace with positive orientation to the region with negative orientation and that the orientation again provides us with a branch 'before' the classical singularity.

For Bianchi models other than Bianchi I, however, the classical approach to the singularity is more complicated than the simpler Kasner behavior. This is most dramatic in the Bianchi IX model where different Kasner epochs succeed each other infinitely many times. It turns out, as we will discuss in the next section, that the classical approach to singularities is modified at small scales by quantum geometry effects which is expected to lead to a simpler picture. 


\section{E. Quantization Choices}

Even after imposing the condition of local stability the constraint expression is far from being unique. There may be other choices for the kinetic term which lead to a locally stable behavior, but they would have to be very special and difficult to find. The advantage of the quantization presented here is that it is rather simple and provides a general formula for all diagonal Bianchi class A models at once without having to find an expression in each case separately.

As for factor ordering ambiguities, the main issue is that of ordering the commutator to the right or to the left. This ordering is the same here as in the Bianchi I case and also in the full theory. Thus, the special issues one has to deal with in models with nonvanishing intrinsic curvature do not change this result. Since the non-singular evolution depends significantly on this kind of ordering, it is robust. The main purpose of this paper is to prove the existence of one quantization for all models in our class which has the correct semiclassical limit, results in a locally stable evolution and is also non-singular in the quantum regime. That such a general quantization fulfilling all the different requirements exists is a non-trivial fact and provides a further check of the methods employed in the full theory.

The second issue, besides the singularity-free evolution, which is robust under the choices we have is the semiclassical behavior and corrections to it. The main effects in this context are insensitive to factor ordering and other choices. As we will discuss in the next section, there are non-perturbative effects coming from the potential term which lead to drastic differences to the classical behavior. Since they come from the potential term and are based on general features of quantizations of inverse triad components, they have to be present in any quantization.

\section{MODIFIED BIANCHI IX BEHAVIOR}

The quantization (26) of the Hamiltonian constraint yields consistent quantizations of the dynamics of Bianchi class A models which in the limit of large triad components reproduce the correct classical behavior. At finite values of $p^{I}$, however, there are always correction terms to the classical equations of motion (13) which can change the classical evolution. It 
is possible to derive different types of corrections and to include them in effective classical equations of motion. There are the following sources of correction terms: First, we can keep more terms beyond the leading order in a Taylor expansion of difference operators which results in a higher order Wheeler-DeWitt equation. These correction terms would correspond to higher curvature terms in an effective action. The second source is the noncommutativity of the $A$-holonomy $h_{I}(A)$ and the $\Gamma$-holonomy $h_{I}(\Gamma)$ in (26). Since we have, for example, $\left\{c_{1}, \Gamma_{1}\right\}=\gamma \kappa p^{2} p^{3}\left(p^{1}\right)^{-3} n^{1}$ which in a quantization would appear as the diagonal operator

$$
i \hbar \Lambda_{1}\left\{\widehat{c_{1}, \Gamma_{1}}\right\}=-h_{1}(A)\left[h_{1}(A)^{-1}, \hat{\Gamma}_{1}\right],
$$

the coefficients of the Wheeler-DeWitt equation receive corrections of the order $o\left(\ell_{\mathrm{P}}^{2} / p^{I}\right)$. Thirdly, we have to use well-defined inverse metric operators when we quantize the spin connection components in (26) which leads to a modified behavior at small scales (as in the isotropic case where it implies inflation [22]).

Since the last effect is non-perturbative while the first two are perturbative (in $\gamma$ ), it can be expected to have the most dramatic consequences. Therefore, we will focus on this type of correction in our discussion of a modified approach to classical singularities. As in 22], we exploit the fact that there are quantization ambiguities [23] which can extend the range of a modified classical behavior [19]. If we choose the ambiguity parameter large enough it is reasonable to use effective classical equations of motion to describe the trajectory of a quantum wave packet while still experiencing the modified behavior at small scales. In particular, the spin connection components will always decrease when we approach the classical singularity close enough whereas they always have some diverging components in the purely classical scenario. The diverging components lead to infinitely high walls in the potential (14) on minisuperspace and to an infinite number of reflections in the Bianchi IX model leading to chaotic behavior [15]. With the modified spin connection, the walls do not grow arbitrarily high but instead shrink once a small triad component is reached. Consequently, one expects that the reflections will cease after a finite number of times and the wave packet will simply move through the classical singularity in a final Kasner epoch.

Here, we will make this explicit by deriving the potential and its changes due to the modified spin connection. A detailed analysis of the modified approach to the singularity will be presented elsewhere [24, 25].

The quantized spin connection operators $\hat{\Gamma}_{I}$ have been defined in equation (28) with the 


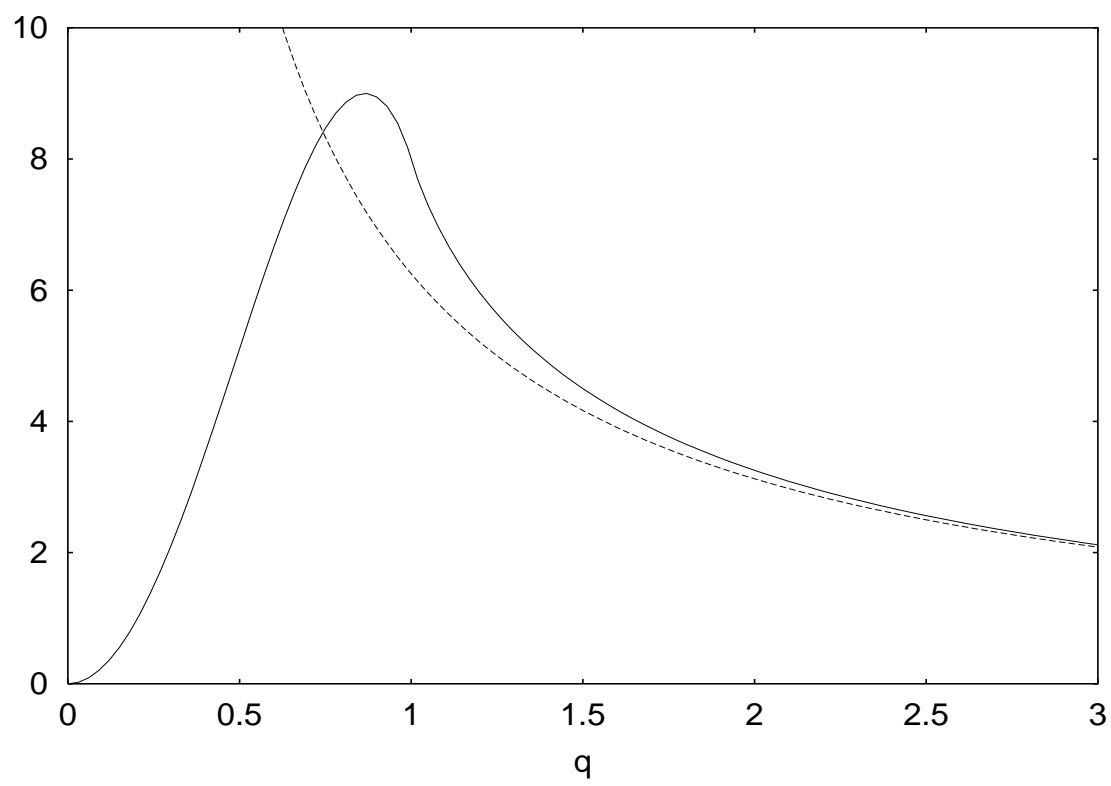

FIG. 1: The function $F(q)$ (solid line) compared to its large- $q$ limit $q^{-1}$ (dashed).

inverse triad operators defined in equation (24). The functions $f_{j}(m)$ can be approximated (with an accuracy increasing with $j$ ) in a manner similar to that discussed in [19]. In terms of $q:=\frac{m}{2 j}$, one obtains

$$
\begin{aligned}
& f_{j}(2 j q) \approx \frac{2}{9} j^{5} F(q) \quad \text { where, } \\
& F(q) \quad:=\frac{4}{25}\left\{3\left[(q+1)^{\frac{5}{2}}-|q-1|^{\frac{5}{2}}\right]-5 q\left[(q+1)^{\frac{3}{2}}-\operatorname{sgn}(q-1)|q-1|^{\frac{3}{2}}\right]\right\}^{2} \\
& \sim q^{-1} \quad \text { for } q \gg 1 \\
& \sim 4 q^{2} \quad \text { for } q \ll 1
\end{aligned}
$$

(see Fig. 11). Limiting behaviors for the eigenvalues of $\widehat{\left(p^{I}\right)_{j}^{-1}}$ are

$$
\begin{aligned}
\left(p^{I}\right)_{j}^{-1}\left(m_{J}\right) & \approx\left(\gamma \ell_{p}^{2} j\right)^{-1} F\left(m_{I} / 2 j\right) \operatorname{sgn}\left(m_{I}\right) & & \\
& \sim p^{I}\left(m_{J}\right)^{-1} & & \text { for }\left|p^{I}\left(m_{J}\right)\right| \gg \gamma \ell_{\mathrm{P}}^{2} j \\
& \sim 4\left(\gamma \ell_{\mathrm{P} j}^{2} j\right)^{-3} p^{I}\left(m_{J}\right)^{2} \operatorname{sgn}\left(m_{I}\right) & & \text { for }\left|p^{I}\left(m_{J}\right)\right| \ll \gamma \ell_{\mathrm{P}}^{2} j
\end{aligned}
$$

Here $p^{I}\left(m_{J}\right)=\frac{1}{2} \gamma \ell_{p}^{2} m_{I}$ are the eigenvalues of $\hat{p}^{I}$. The eigenvalues of the spin connection operators are obtained as,

$$
\Gamma_{I}^{(j)}(\vec{m}) \approx \frac{1}{4 j}\left[\quad n^{J} m_{K} F\left(m_{J} / 2 j\right) \operatorname{sgn}\left(m_{J}\right)+n^{K} m_{J} F\left(m_{K} / 2 j\right) \operatorname{sgn}\left(m_{K}\right)\right.
$$




$$
\begin{aligned}
& \left.-n^{I} m_{J} m_{K} F^{2}\left(m_{I} / 2 j\right) / 2 j\right] \\
\sim & \frac{1}{2}\left[n^{J} \frac{p^{K}\left(m_{K}\right)}{p^{J}\left(m_{J}\right)}+n^{K} \frac{p^{J}\left(m_{J}\right)}{p^{K}\left(m_{K}\right)}-n^{I} \frac{p^{J}\left(m_{J}\right) p^{K}\left(m_{K}\right)}{\left(p^{I}\right)^{2}\left(m_{I}\right)}\right] \quad\left(\frac{\left|p^{I}\right|}{\gamma \ell_{\mathrm{P}}^{2} j} \gg 1\right) \\
\sim & \frac{1}{4 j^{3}}\left[n^{J} m_{K} m_{J}^{2} \operatorname{sgn}\left(m_{J}\right)+n^{K} m_{J} m_{K}^{2} \operatorname{sgn}\left(m_{K}\right)-n^{I} \frac{m_{J} m_{K} m_{I}^{4}}{2 j^{3}}\right] \\
\approx & 2\left(\gamma \ell_{p}^{2} j\right)^{-3} p^{J}\left(m_{J}\right) p^{K}\left(m_{K}\right)\left[n^{J}\left|p^{J}\left(m_{J}\right)\right|+n^{K}\left|p^{K}\left(m_{K}\right)\right|\right] \quad\left(\frac{\left|p^{I}\right|}{\gamma \ell_{\mathrm{P}}^{2} j} \ll 1\right)
\end{aligned}
$$

In the last equation we have dropped the sub-leading third term. Evidently, the eigenvalues of the spin connection operators approach their classical expressions for large values of the triad components while for small values the quantum modification shows up. To find the potential in this regime, we must use the first line of eqn. (14) since the second follows by using the classical expression for $\Gamma_{I}$. For $\left|p^{I}\right| \ll \gamma \ell_{\mathrm{P}}^{2} j$ this leads to,

$$
\begin{aligned}
W_{j}(\vec{p}):= & 2 p^{1} p^{2}\left(\Gamma_{1}^{(j)} \Gamma_{2}^{(j)}-n^{3} \Gamma_{3}^{(j)}\right)+\text { cyclic } \\
\approx & {\left[8\left(\gamma \ell_{p}^{2} j\right)^{-6}\left(p^{1} p^{2} p^{3}\right)^{2}\left(n^{2}\left|p^{2}\right|+n^{3}\left|p^{3}\right|\right)\left(n^{1}\left|p^{1}\right|+n^{3}\left|p^{3}\right|\right)\right) } \\
& \left.-4\left(\gamma \ell_{p}^{2} j\right)^{-3}\left(p^{1} p^{2}\right)^{2} n^{3}\left(n^{1}\left|p^{1}\right|+n^{2}\left|p^{2}\right|\right)\right]+ \text { cyclic }
\end{aligned}
$$

Dropping the $o\left(j^{-6}\right)$ term compared to the $o\left(j^{-3}\right)$, for $\left|p^{I}\right| \ll \gamma \ell_{p}^{2} j$, we get

$$
\begin{gathered}
W_{j}(\vec{p}) \approx-4\left(\gamma \ell_{p}^{2} j\right)^{-3}\left[n^{1} n^{2}\left(p^{3}\right)^{2}\left(\left|p^{1}\right|^{3}+\left|p^{2}\right|^{3}\right)+n^{2} n^{3}\left(p^{1}\right)^{2}\left(\left|p^{2}\right|^{3}+\left|p^{3}\right|^{3}\right)\right. \\
\left.+n^{3} n^{1}\left(p^{2}\right)^{2}\left(\left|p^{3}\right|^{3}+\left|p^{1}\right|^{3}\right)\right]
\end{gathered}
$$

By contrast, for $\left|p^{I}\right| \gg \gamma \ell_{p}^{2} j$, the potential takes the form,

$$
\begin{aligned}
W_{j}(\vec{p}) \approx \frac{1}{2}[ & \left\{p^{1} p^{2}\left(p^{3}\right)^{-1} n^{3}\right\}^{2}+\left\{p^{2} p^{3}\left(p^{1}\right)^{-1} n^{1}\right\}^{2}+\left\{p^{3} p^{1}\left(p^{2}\right)^{-1} n^{2}\right\}^{2} \\
& \left.-2\left\{\left(p^{1}\right)^{2} n^{2} n^{3}+\left(p^{2}\right)^{2} n^{3} n^{1}+\left(p^{3}\right)^{2} n^{1} n^{2}\right\}\right]
\end{aligned}
$$

Specializing to Bianchi-IX this is:

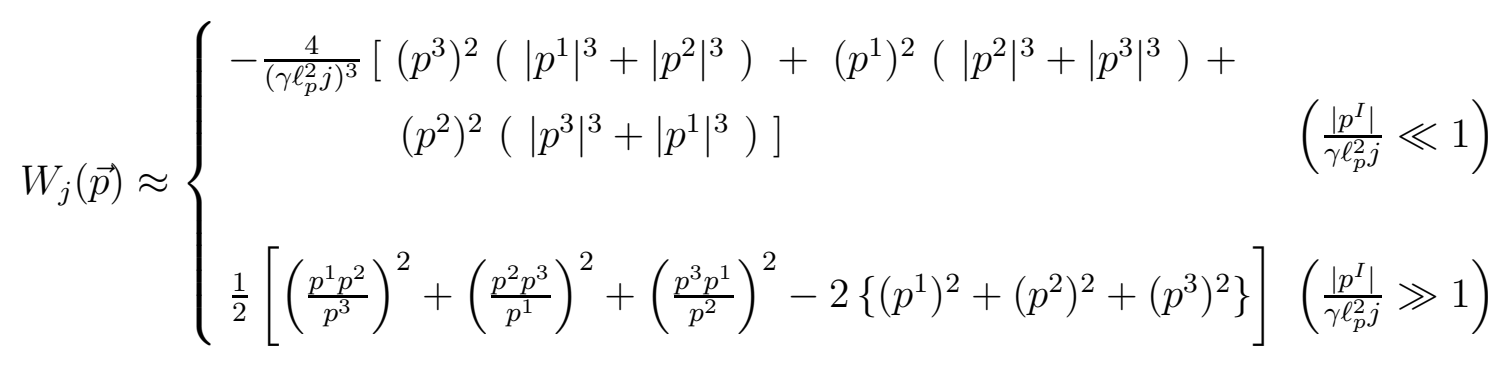




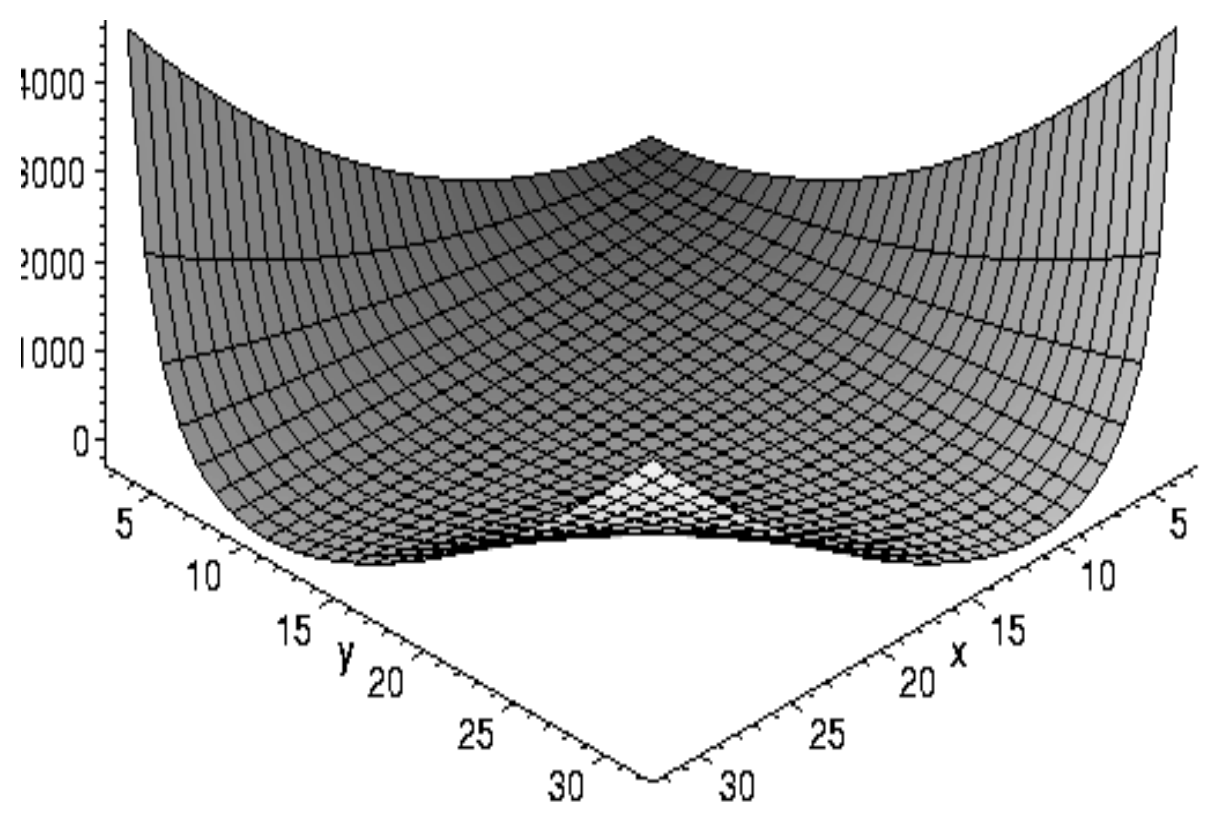

FIG. 2: Classical potential as a function of $x:=2 p^{1} / \gamma \ell_{\mathrm{P}}^{2}$ and $y:=2 p^{2} / \gamma \ell_{\mathrm{P}}^{2}$ with $2 p^{3} / \gamma \ell_{\mathrm{P}}^{2}=10$.

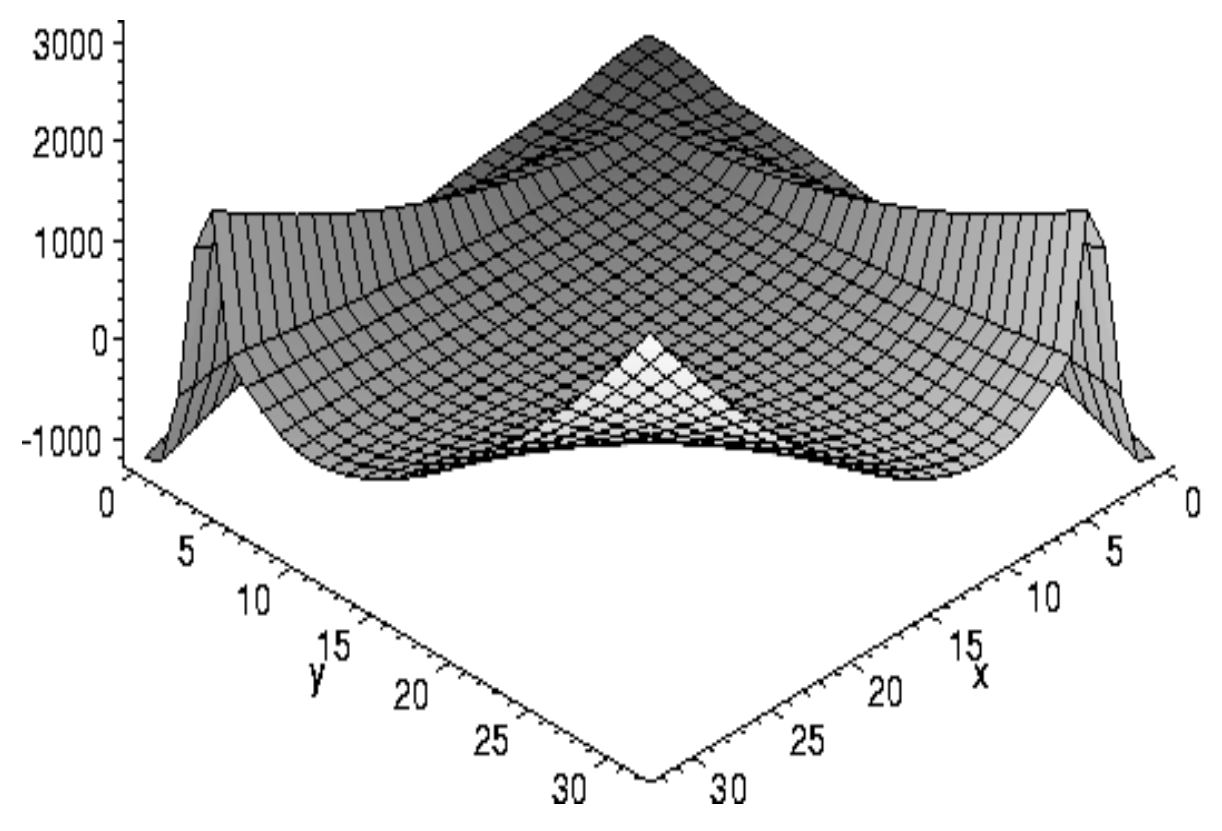

FIG. 3: Modified potential with collapsed walls at small $p^{1}$ and $p^{2}$ (with $j=3$ ).

A sample snapshot of the modified potential is shown in Figs. 3 and 4 together with the classical potential (Fig. 2) for comparison. With the modified potential, the classical 


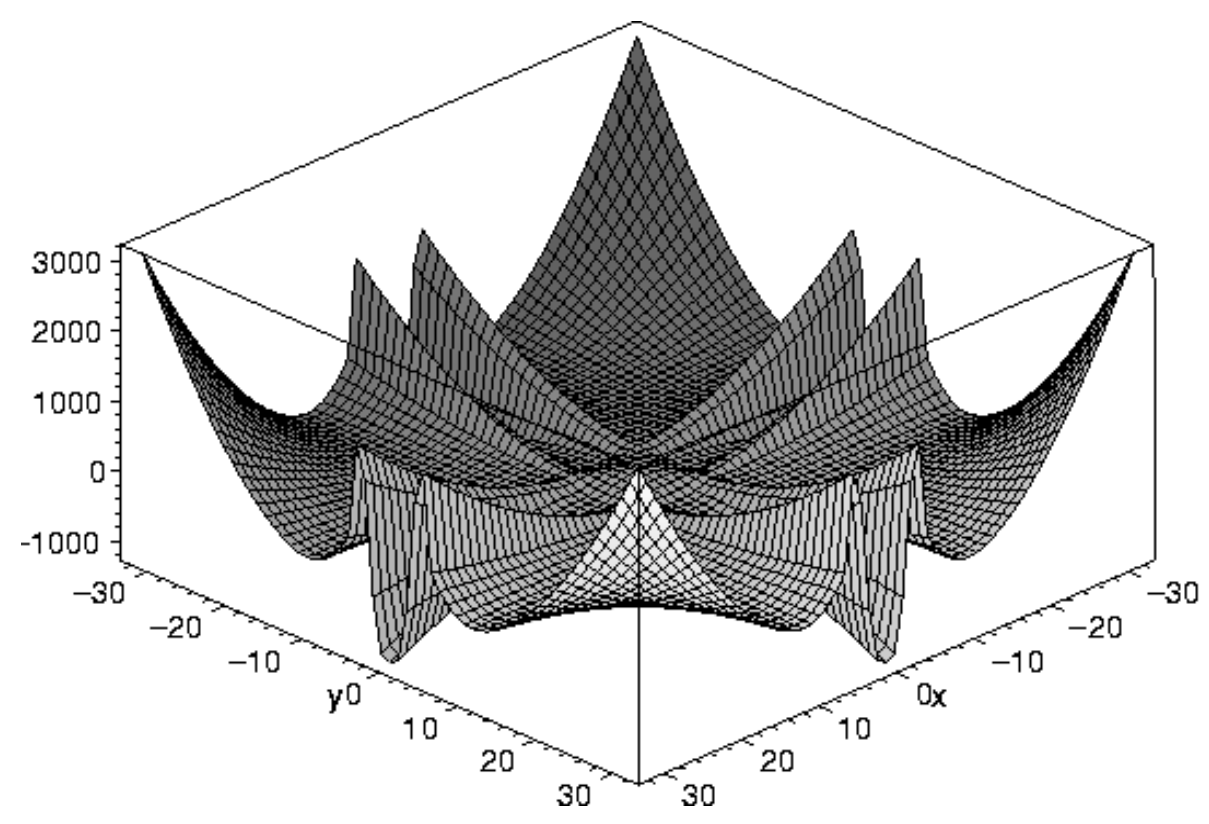

FIG. 4: Modified potential with collapsed walls at small $p^{1}$ and $p^{2}$ (diagonal canyons) with $j=3$. Also regions for negative $p^{I}$ are shown to which a test particle in the potential can move; with the original potential only the lower quadrant is allowed.

Hamiltonian (10) becomes

$$
\kappa N H_{j}^{\mathrm{eff}}\left(\pi_{I}, q^{I}\right)=-\frac{1}{2}\left(\pi_{1} \pi_{2}+\pi_{2} \pi_{3}+\pi_{3} \pi_{1}\right)+W_{j}\left(e^{2 q^{1}}, e^{2 q^{2}}, e^{2 q^{3}}\right)
$$

We have thus derived an effective potential modified by the non-perturbative quantum effects. Other correction terms, such as higher order contributions to the kinetic part coming from the discreteness, are perturbative and less important as remarked earlier. Note that classical regime is identified by $m_{I}=p^{I}\left(\frac{\gamma \ell_{\mathrm{P}}^{2}}{2}\right)^{-1} \gg 1$. The quantum modifications however are dominant in the small $q=\frac{m}{2 j}$ regimes. Choosing larger values of the ambiguity parameter $j$, thus allows us to access the quantum effects even while staying in the classical regime. We can thus study the qualitative effects of quantum modifications within a classical framework by using the effective potential instead of using a fully quantum description in terms of a wave function on minisuperspace. Effective classical equations of motion, in terms of an external time parameter, can be derived from the Hamiltonian (82) as we did in Section \A. The corresponding classical trajectories can be thought of as (approximately) describing the motion of a quantum wave packet, giving suggestions regarding the full quantum behavior.

Alternatively, one can also analyze the modified behavior in terms of an internal time 

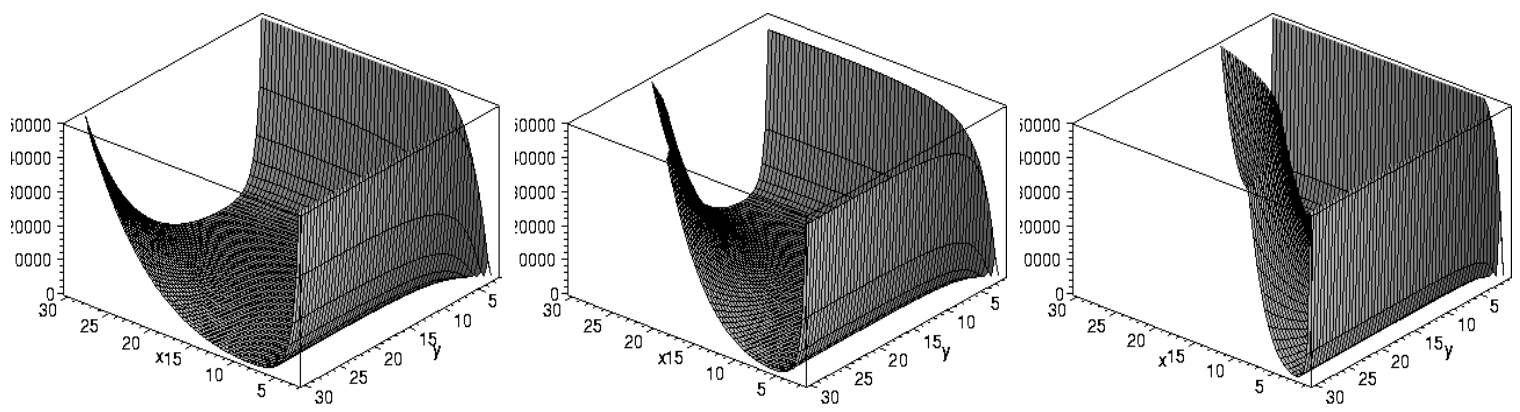

FIG. 5: Classical potential at constant volume $V_{1}=\left(\frac{13}{2} \gamma \ell_{\mathrm{P}}^{2}\right)^{\frac{3}{2}}, V_{2}=\left(5 \gamma \ell_{\mathrm{P}}^{2}\right)^{\frac{3}{2}}$, and $V_{3}=\left(3 \gamma \ell_{\mathrm{P}}^{2}\right)^{\frac{3}{2}}$ (from left to right). While moving in the triangular valley the universe is pushed toward the classical singularity $(x=y=0)$ by reflections on the moving left wall.
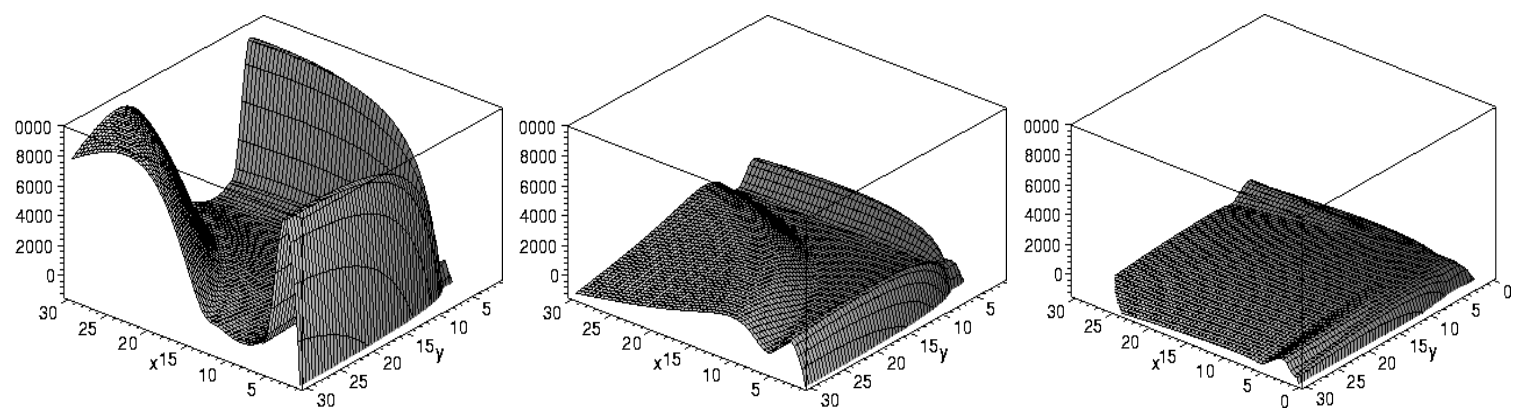

FIG. 6: Modified potential with collapsing walls (for $j=3$ ) at constant volume $V_{1}=\left(\frac{13}{2} \gamma \ell_{\mathrm{P}}^{2}\right)^{\frac{3}{2}}$, $V_{2}=\left(5 \gamma \ell_{\mathrm{P}}^{2}\right)^{\frac{3}{2}}$, and $V_{3}=\left(3 \gamma \ell_{\mathrm{P}}^{2}\right)^{\frac{3}{2}}$ (from left to right).

namely the volume. The Hamiltonian (82) is in fact hyperbolic. The potential now has a non-factorizable time dependence and the analysis of motion is more complicated. Figs. [5] and [6] show the classical and the modified potential, respectively, at fixed volume $\sqrt{\left|p^{1} p^{2} p^{3}\right|}$ rather than at fixed $p^{3}$ as in Figs. 2] and 3. One can already expect from the figures that the behavior will change dramatically once the universe reaches a small triad component. For a detailed analysis see [24, 25].

\section{CONCLUSIONS}

In this paper we have extended the methods of homogeneous loop quantum cosmology to models with non-zero spin connection. These models have non-trivial intrinsic curvature from the symmetric background which requires a special treatment compared to the Bianchi 
I model as well as compared to the full theory. Initially, therefore, part of the guidance from the full theory is lost and there are more ambiguities when one quantizes the Hamiltonian constraint.

In 11] two conditions which have to be imposed for a reasonable quantization, have been introduced and discussed in detail. These stem primarily from the requirement that the loop quantization admit physical states which, in a semiclassical regime, have much less sensitivity to variations on the Planck scale so that the idealization of the continuum geometric formulation is well justified. This has been made more explicit by the formulation of the continuum approximation.

In Section [II] we introduced a quantization of the Hamiltonian constraint for all diagonalized Bianchi class A models, and showed that the two conditions are satisfied. It was important to take the freedom of phases in the triad representation into account which we did by transforming to a new wave function $\tilde{s}$ in (30). The transformation was dictated by the requirement of reproducing the Wheeler-DeWitt equation as the leading approximation. It is satisfying to note that the same transformation also appeared in [26] where it was used in the context of relating the wave functions obtained in the Schrödinger quantization based on connection variables and the wave functions of the Wheeler-DeWitt quantization based on the metric variables. Since the two sets of basic variables differ significantly only when the spin connection is non-zero which happens when the intrinsic curvature is non-zero, nontrivial phases show up in this situation. The same transformation then appears whether one uses a loop quantization or a Schrödinger quantization in the connection formulation and compares with the Wheeler-DeWitt quantization. We also showed that the mechanism for the absence of singularities continues to hold, despite the phases, exactly as in the case of the Bianchi-I model [6]. Thus, as the main result of this paper we proved that for all diagonal Bianchi class A models there is a loop quantization which satisfies all the conditions for a good continuum limit as well as that of a singularity-free evolution. That such a quantization exists at all, and even one which provides a general form for all the models, is a non-trivial fact and gives further credence to the viability of loop methods and the physical applications obtained so far.

In section $\llbracket$ we carried out a preliminary exploration of the consequences of the quantized spin connection and the correspondingly modified potential. We focused on the Bianchi IX case and pointed out that unlike the classical potential which has infinite walls as the 
singularity is approached, the quantum modified potential has finite walls. Since the infinite walls are responsible for the chaotic approach to the singularity, we expect that the finite walls of the modified potential will significantly alter the approach to the classical singularity [24, 25].

Finally a remark about the relevance of the results is in order. Since the connection of the quantization discussed here to the full theory is weaker, it is legitimate to question what this quantization may have to do with the full theory. Firstly, we observe that the methods adopted as well as the two admissibility criteria work uniformly for all homogeneous models (including isotropic sub-models) which do not have a non-zero spin connection. The direct loss of connection to the full theory is only in the models with spin connection. The treatment of the spin connection enters in two distinct places, firstly in using the inverses of triad components and secondly in the modification of holonomies. The first one is quite natural and inherits the ambiguities in the definition of inverses [19]. The second one has additional ambiguities due to factor ordering. It is already clear that the first type of ambiguity is not fixed by the two admissibility criteria. Our emphasis in this work has been to demonstrate the existence of a uniform method of quantization which satisfies the two admissibility criteria and is singularity free. The uniqueness type of issues, while important, requires a systematic classification of possible ambiguities and is beyond the scope of the present work. Likewise the general question of how many of the results of a minisuperspace quantization can survive the full theory is also beyond the scope of this paper.

While the spin connection is seen to require a careful treatment of the continuum approximation and also to lead to a modified approach to the BKL singularity in the context of homogeneous models, its role in the full theory is more complicated and is an open issue. For instance, the phase factor in $\tilde{s}$ which has the form,

$$
\exp \left(i \gamma^{-1} \ell_{\mathrm{P}}^{-2} p^{I} \Gamma_{I}\right)=\exp \left(i \gamma^{-1} \ell_{\mathrm{P}}^{-2} \int \mathrm{d}^{3} x E_{i}^{a} \Gamma_{a}^{i}\right)
$$

is not even well-defined in the full theory due to the transformation properties of the spin connection (which is no longer a covariant object). Since the spin connection can be made arbitrarily small locally, by choosing appropriate coordinates, one can expect that the diffeomorphism constraint and its algebra with the Hamiltonian constraint will play a role in this issue. Requirements such as the local stability condition can also be expected to play a role in analyzing this issue, at least with a local version in the absence of a global internal 
time.

\section{Acknowledgments}

M. B. and G. D. are grateful to Madhavan Varadarajan and Naresh Dadhich for invitations to and hospitality at the RRI, Bangalore, and IUCAA, Pune, where part of the work has been done. M. B. also thanks the IMSC, Chennai, for hospitality. The work of M. B. and K. V. was supported in part by NSF grant PHY00-90091 and the Eberly research funds of Penn State.

[1] Misner C W 1969 Quantum Cosmology. I Phys. Rev. 186 1319-1327

[2] Bojowald M 2000 Loop Quantum Cosmology: I. Kinematics Class. Quantum Grav. 17 14891508

[3] Bojowald M 2000 Loop Quantum Cosmology: II. Volume Operators Class. Quantum Grav. 17 1509-1526

[4] Bojowald M 2001 Loop Quantum Cosmology III: Wheeler-DeWitt Operators Class. Quantum Grav. 18 1055-1070

[5] Bojowald M 2001 Loop Quantum Cosmology IV: Discrete Time Evolution Class. Quantum Grav. 18 1071-1088

[6] Bojowald M 2003 Homogeneous loop quantum cosmology Class. Quantum Grav. 20 2595-2615

[7] Thiemann T 1998 Quantum Spin Dynamics (QSD) Class. Quantum Grav. 15 839-873

[8] Bojowald M 2002 Isotropic Loop Quantum Cosmology Class. Quantum Grav. 19 2717-2741

[9] Bojowald M 2001 Absence of a Singularity in Loop Quantum Cosmology Phys. Rev. Lett. 86 $5227-5230$

[10] Belinskii V A, Khalatnikov I M, and Lifschitz E M 1982 A general solution of the Einstein equations with a time singularity Adv. Phys. 13 639-667

[11] Bojowald M and Date G 2004 Consistency Conditions for Fundamentally Discrete Theories Class. Quantum Grav. 21 121-143

[12] Ashtekar A, Baez J C, Corichi A, and Krasnov K 1998 Quantum Geometry and Black Hole Entropy Phys. Rev. Lett. 80 904-907 
[13] Ashtekar A, Baez J C, and Krasnov K 2001 Quantum Geometry of Isolated Horizons and Black Hole Entropy Adv. Theor. Math. Phys. 4 1-94

[14] Misner, C W 1969 Mixmaster Universe Phys. Rev. Lett. 22 1071-1074

[15] Hobill D, Burd A, and Coley A 1994 Deterministic chaos in general relativity (New York: Plenum Press)

[16] Ashtekar A, Bojowald M, and Lewandowski, J 2003 Mathematical structure of loop quantum cosmology Adv. Theor. Math. Phys. 7 233-268

[17] Thiemann T 1998 QSD V: Quantum Gravity as the Natural Regulator of Matter Quantum Field Theories Class. Quantum Grav. 15 1281-1314

[18] Bojowald M 2001 Inverse Scale Factor in Isotropic Quantum Geometry Phys. Rev. D 64 084018

[19] Bojowald M 2002 Quantization ambiguities in isotropic quantum geometry Class. Quantum Grav. 19 5113-5130

[20] Bojowald M and Vandersloot K 2003 Loop quantum cosmology, boundary proposals, and inflation Phys. Rev. D 67124023

[21] Bojowald M 2001 The Semiclassical Limit of Loop Quantum Cosmology Class. Quantum Grav. 18 L109-L116

[22] Bojowald M 2002 Inflation from quantum geometry Phys. Rev. Lett. 89261301

[23] Gaul M and Rovelli C 2001 A generalized Hamiltonian Constraint Operator in Loop Quantum Gravity and its simplest Euclidean Matrix Elements Class. Quantum Grav. 18 1593-1624

[24] Bojowald M and Date G 2003 Quantum suppression of the generic chaotic behavior close to cosmological singularities e-print gr-qc/0311003, to appear in Phys. Rev. Lett.

[25] Bojowald M, Date G and Hossain G M 2003 The Bianchi IX model in loop quantum cosmology Preprint in preparation

[26] Kodama H 1988 Specialization of Ashtekar's Formalism to Bianchi Cosmology Prog. Theor. Phys. 80 1024-1040 